CERN-TH.6961/93

\title{
SUPERSYMMETRIC CALCULATION OF MIXED KÄHLER-GAUGE AND MIXED KÄHLER-LORENTZ ANOMALIES
}

\author{
Gabriel Lopes Cardoso' \\ and \\ Burt A. Ovruth \\ Theory Division, CERN, CH-1211 Geneva 23, Switzerland
}

\begin{abstract}
We present a manifestly supersymmetric procedure for calculating the contributions from matter loops to the mixed Kähler-gauge and to the mixed Kähler- Lorentz anomalies in $N=1, D=4$ supergravity-matter systems. We show how this procedure leads to the well-known result for the mixed Kähler-gauge anomaly. For general supergravity-matter systems the mixed Kähler-Lorentz anomaly is found to contain a term proportional to $\mathcal{R}^{2}$ with a background field dependent coefficient as well as terms proportional to $\left(C_{m n p q}\right)^{2}$ and to the Gauss-Bonnet topological density. We briefly comment on the relationship between the mixed Kähler-Lorentz anomaly and the moduli dependent threshold corrections to gravitational couplings in $Z_{N}$ orbifolds.
\end{abstract}

CERN-TH.6961/93

August 1993

\footnotetext{
${ }^{1}$ Work supported in part by the Department of Energy under Contract No. DOE-AC02-76-ERO3071.

${ }^{2}$ On sabbatical leave from the Department of Physics, University of Pennsylvania, Philadelphia, PA 19104-6396, USA.
} 


\section{Introduction}

As it is well known [14, 15], the tree-level Lagrangian describing the coupling of chiral and antichiral matter supermultiplets $\Phi$ and $\Phi^{\dagger}$ to $N=1, D=4$ supergravity is specified by three fundamental functions, namely the Kähler potential $K$, the holomorphic superpotential $W$ and the holomorphic gauge coupling function $f$. In the conventional formulation of the theory, the tree-level Lagrangian is invariant under combined super-Weyl-Kähler transformations [14, 15]. These consist of superfield rescalings of the supervielbein, which leave the conventional torsion constraints invariant, followed by Kähler transformations of the three fundamental functions $K, W, f$. The chiral matter superfields do not transform under either the super-Weyl or the Kähler transformations. On the other hand, the tree-level Lagrangian in the conventional formulation has, in general, a non-canonical gravitational kinetic energy term of the form $h(A, \bar{A}) \mathcal{R}$, where $A$ represents component matter scalar fields, $h$ is some calculable function and $\mathcal{R}$ is the curvature scalar. It is also well known [14, 15] that, by appropriate rescaling of the graviton and of the gravitino as well as of the auxiliary fields $M$ and $b_{a}$ of minimal supergravity, one can obtain a new Lagrangian with appropriately Einstein normalized gravitational kinetic energy, $\mathcal{R}$. This rescaling can either be performed at the component field level [14, 15] or at the superfield level [5, 6, 7], the latter having the advantage of being manifestly supersymmetric. It turns out that this rescaled Lagrangian is invariant under an important new symmetry, called Kähler symmetry [14, 15, 团. By explicitly adding the Kähler transformations to the structure group of superspace [5, 6, 7], one can give a complete superfield description of this appropriately Einstein normalized supergravity-matter theory. The new underlying superspace is called Kähler superspace. The Kähler invariance of the Einstein normalized tree-level Lagrangian can be traced back to the super-Weyl-Kähler invariance of the tree-level Lagrangian in the conventional superspace formulation of the theory [14, 15, 31]. Hence, a chiral matter superfield in Kähler superspace carries a zero Kähler charge. Its fermionic component field, however, carries a non-vanishing Kähler charge and, consequently, couples to the component Kähler connection $a_{a}$, which is a composite connection made out of component matter fields. Other fermions, such as gauginos and the gravitino, also carry a non-vanishing Kähler charge and, hence, also couple 
to the Kähler connection $a_{a}$. As always in four dimensions, the Kähler symmetry of the Einstein normalized tree-level Lagrangian might get spoiled by one-loop component triangle graphs with fermions running in the loop and with at least one Kähler connection sticking out. This is, in fact, what happens in general supergravity-matter theories [16, 9, 10, 26]. On the other hand, since only fermions carry Kähler charge, it is impossible to construct component graphs having legs of the Kähler type sticking out and having component fields running in the loop other than fermions. This, however, creates a puzzle. Namely, it would appear that it is then impossible to construct a supersymmetric expression for the component Kähler anomaly in Kähler superspace because of missing component graphs.

In this paper, we will present a manifestly supersymmetric procedure for calculating mixed Kähler-gauge and mixed Kähler-Lorentz anomalies. These are the ones generated by component fermionic triangle graphs with only one Kähler connection sticking out. These mixed Kähler anomalies have important physical consequences. They have been shown to lead to threshold corrections to gauge and gravitational couplings [16, 9, 10, 26, 18, 2, 2, 25] in string theory as well as to inflationary supergravity models [11, 12]. We will restrict our discussion to the contribution from matter loops to these mixed anomalies. We believe, though, that the procedure described in this paper can also be extended to include contributions from gauge and gravitational fields running in the loop.

The origin of the puzzle is not hard to understand. As mentioned above, a chiral matter superfield carries vanishing Kähler charge $\omega$ in Kähler superspace. This means that there is no direct coupling $\Phi^{\dagger} e^{\omega K} \Phi$ of the Kähler prepotential $K$ to quadratic matter superfields, since $\omega(\Phi)=0$. This is to be contrasted with the coupling of the gauge prepotential $V$ to charged matter as $\Phi^{\dagger} e^{V} \Phi$. Were one to examine the pure gauge anomaly three-point function, one would find a supersymmetric result, since it could have been obtained by calculating a superfield triangle graph with $\Phi$ running in the loop and $V$-legs sticking out. It seems then clear that, in order to directly calculate supersymmetric expressions for the mixed Kähler anomalies, one must go to a formalism where $K$ couples to chiral matter as $\Phi^{\dagger} e^{K} \Phi$. The conventional superspace formalism is such a framework. It allows for a superfield prepotential formalism describing the coupling of quantum matter superfields $\phi$ to background prepotentials 
such as $K$. An example of such a coupling is precisely $\phi^{\dagger} e^{-\frac{1}{3} K} \phi$. Since there exists a superfield prepotential formalism, all calculations of anomalous triangle graphs in the conventional formulation can, in principle, be performed at the superfield level, yielding automatically supersymmetric results.

The relevant tree-level symmetry in the conventional superspace formulation is the tree-level super-Weyl-Kähler symmetry. Hence, of relevance in the conventional formulation are the couplings of quantum matter superfields to Kähler as well as supergravity prepotentials, and one has to look out for the breakdown of the tree-level super-WeylKähler symmetry due to the non-vanishing of mixed triangle graphs with quantum matter superfields running in the loop. We do indeed find that such mixed triangle graphs are non-vanishing, and, hence, that they contribute to the breakdown of the super-Weyl-Kähler symmetry. As stated above, results in the conventional superspace formulation of the theory can be transformed over into Kähler superspace by particular superfield rescalings of the underlying torsion constraints of conventional superspace [5, 6, 7]. Upon applying these rescalings to the mixed super-Weyl-Kähler anomalies in conventional superspace, we arrive at manifestly supersymmetric results in Kähler superspace which qualify to be called the supersymmetric mixed Kähler-gauge and the supersymmetric mixed Kähler-Lorentz anomaly, respectively. In this way, we give the first complete calculation for, and rediscover the well-known result [16, 10, 26] for the supersymmetric mixed Kähler-gauge anomaly. We also give the first complete calculation for the supersymmetric mixed Kähler-Lorentz anomaly. When putting the gravitational fields on-shell, the supersymmetric mixed Kähler-Lorentz anomaly calculated using our procedure also reduces to its well-known form given in [9, 10]. Off-shell, however, it contains an additional term proportional to $\mathcal{R}^{2}$ which is not part of the Gauss-Bonnet topological density. Two remarks concerning this term need to be made. First of all, this $\mathcal{R}^{2}$-term, present in general supergravity-matter theories, arises from graphs constructed out of vertices of the type $E e^{-\frac{1}{3} K} \phi^{2}$ in the conventional formulation. Such vertices are not invariant under conformal transformations (these are defined in section 6), and, hence, the growing of a term proportional to $\mathcal{R}^{2}$ not contained in the Gauss-Bonnet combination is not forbidden by any symmetry argument. Secondly, the coefficient of this $\mathcal{R}^{2}$ term comes out to be background field dependent.

We close this paper with a remark on the relationship between the supersymmetric 
mixed Kähler-Lorentz anomaly, as computed in this paper, and the moduli dependent threshold corrections to gravitational couplings in $Z_{N}$ orbifolds computed in [3]. These threshold corrections turn out to be proportional to the trace anomaly coefficients of the different fields coupled to gravity. From the field theory analysis presented in this paper this can be understood by noticing that there are two distinct terms which contribute to the Kähler anomaly. One of them comes with a coefficient proportional to the trace anomaly whereas the other one comes with a coefficient proportional to the chiral anomaly in conventional superspace. It is this latter contribution which gets removed by the Green-Schwarz mechanism [16, 9, 10], yielding threshold corrections proportional to the trace anomaly coefficients of the various fields coupled to gravity.

\section{2. $U_{K}(1)$ Superspace and Tree-Level Symmetries}

In this section, we will briefly review some of the features of Kähler superspace geometry which will be relevant in the subsequent discussion. A complete description of the properties of $U_{K}(1)$ superspace can be found in [7]. The structure group of Kähler superspace is taken to be $S L(2, C) \times U_{K}(1)$ and, accordingly, one introduces two Lie algebra valued one-form gauge connections $\phi_{B}{ }^{A}=d z^{M} \phi_{M B}{ }^{A}$ and $A=d z^{M}$ $A_{M}$ corresponding to the Lorentz and $U_{K}(1)$ groups, respectively. In addition, one introduces a supervielbien $E_{M}{ }^{A}$ and the associated one-forms $E^{A}=d z^{M} E_{M}{ }^{A}$. The $U_{K}(1)$ gauge connection $A$ is a composite gauge connection defined by

$$
\begin{aligned}
A_{\alpha} & =\frac{1}{4} \mathcal{D}_{\alpha} K \\
A^{\dot{\alpha}} & =-\frac{1}{4} \overline{\mathcal{D}}^{\dot{\alpha}} K \\
A_{\alpha \dot{\alpha}} & =-\frac{i}{8}\left[\mathcal{D}_{\alpha}, \overline{\mathcal{D}}_{\dot{\alpha}}\right] K
\end{aligned}
$$

where the prepotential $K\left(\Phi_{i}, \Phi_{i}^{\dagger}\right)$ is the Kähler potential for matter chiral superfields $\Phi_{i}$. All matter superfields have vanishing $U_{K}(1)$ weight, $\omega_{K}\left(\Phi_{i}\right)=0$. Under a Kähler transformation

$$
\kappa^{2} K\left(\Phi_{i}, \Phi_{i}^{\dagger}\right) \rightarrow \kappa^{2} K\left(\Phi_{i}, \Phi_{i}^{\dagger}\right)+F\left(\Phi_{i}\right)+\bar{F}\left(\Phi_{i}{ }^{\dagger}\right)
$$


the one-form $A$ transforms as

$$
A \rightarrow A+\kappa^{-2} \frac{i}{2} d \operatorname{Im} F
$$

where $\operatorname{Im} F=\frac{F-\bar{F}}{2 i}$ and $\kappa^{2}=8 \pi M_{P}^{-2} . M_{P}$ is the Planck mass. Also, under a Kähler transformation the supervielbein one-forms $E^{A}$ can be shown [7] to transform as

$$
E^{A} \rightarrow E^{A} \exp \left[-\frac{i}{2} \omega\left(E^{A}\right) \operatorname{Im} F\right]
$$

where

$$
\omega\left(E^{\alpha}\right)=1, \omega\left(E_{\dot{\alpha}}\right)=-1 \omega\left(E^{a}\right)=0 .
$$

Solving the Bianchi identities subject to a set of constraints [28], one finds that all components of the torsion and curvature may be expressed in terms of a set of superfields and their coordinate derivatives:

$$
\begin{array}{lccccc}
\text { superfield } & R & R^{\dagger} & G_{\alpha \dot{\alpha}} & W_{\alpha \beta \gamma}, X_{\alpha} & \bar{W}_{\dot{\alpha} \dot{\beta} \dot{\gamma}}, \bar{X}_{\dot{\alpha}} \\
U_{K}(1) \text { weight } & 2 & -2 & 0 & 1 & -1
\end{array}
$$

where

$$
\begin{aligned}
& X_{\alpha}=\mathcal{D}_{\alpha} \mathcal{R}-\overline{\mathcal{D}}^{\dot{\alpha}} G_{\alpha \dot{\alpha}}=-\frac{\kappa^{2}}{8}\left(\overline{\mathcal{D}}^{2}-8 R\right) \mathcal{D}_{\alpha} K \\
& \bar{X}^{\dot{\alpha}}=\overline{\mathcal{D}}^{\dot{\alpha}} R^{\dagger}+\mathcal{D}_{\alpha} G^{\alpha \dot{\alpha}}=-\frac{\kappa^{2}}{8}\left(\mathcal{D}^{2}-8 R^{\dagger}\right) \overline{\mathcal{D}}^{\dot{\alpha}} K
\end{aligned}
$$

$X_{\alpha}$ is the superfield fieldstrength of the $U_{K}(1)$ gauge connection. If we further assume that there is an internal gauge group, then we must introduce yet another Lie algebra valued one-form gauge connection $\mathcal{A}_{a}{ }^{b}=d z^{M} \mathcal{A}_{M a}{ }^{b}$. Solving the Bianchi identities now introduces a new superfield fieldstrength, $W_{\alpha}^{a}$, with $U_{K}(1)$ weight $\omega\left(W_{\alpha}^{a}\right)=1$.

Using these superfields, one can write down the tree-level superfield Lagrangian in Kähler superspace [5, 6, 7]. It consists of three parts, each specified by a fundamental and independent function. The first part, specified entirely by the Kähler potential, is the supergravity-matter kinetic energy term given by

$$
\mathcal{L}_{0}=-3 \kappa^{-2} \int d^{4} \theta E[K]
$$

where $E$ is the superdeterminant. The second part, specified by the holomorphic superpotential $W\left(\Phi_{i}\right)$, is the potential energy term given by

$$
\mathcal{L}_{P E}=\frac{1}{2} \int d^{4} \theta \frac{E}{R} e^{\kappa^{2} \frac{K}{2}} W\left(\Phi_{i}\right)+\text { h.c. }
$$


Finally, the Yang-Mills Lagrangian is given by

$$
\mathcal{L}_{Y M}=\frac{1}{8} \int d^{4} \theta \frac{E}{R} f\left(\Phi_{i}\right)_{a b} W^{\alpha a} W_{\alpha}^{b}+\text { h.c. }
$$

where $f\left(\Phi_{i}\right)_{a b}$ is the holomorphic gauge coupling function. The total Lagrangian possesses, at the tree-level, three symmetries.

1. Kähler invariance:

Under Kähler transformation $\kappa^{2} K \rightarrow \kappa^{2} K+F+\bar{F}$, it can be shown [5, 6, 7] that

$$
\begin{aligned}
& E \rightarrow E \\
& R \rightarrow R e^{-(F-\bar{F}) / 2}
\end{aligned}
$$

One also, simultaneously, transforms $W$ and $W_{\alpha}$ as

$$
\begin{aligned}
W & \rightarrow e^{-F} W \\
W_{\alpha} & \rightarrow e^{-(F-\bar{F}) / 4} W_{\alpha} .
\end{aligned}
$$

Then

$$
\mathcal{L}_{0}^{\prime}=-3 \kappa^{-2} \int d^{4} \theta E^{\prime}=-3 \kappa^{-2} \int d^{4} \theta E=\mathcal{L}_{0}
$$

and

$$
\begin{aligned}
\mathcal{L}_{P E}^{\prime} & =\frac{1}{2} \int d^{4} \theta \frac{E^{\prime}}{R^{\prime}} e^{\kappa^{2} K^{\prime} / 2} W^{\prime}+\text { h.c. } \\
& =\frac{1}{2} \int d^{4} \theta \frac{E e^{\kappa^{2} K / 2} W e^{(F+\bar{F}) / 2} e^{-F}}{R e^{-(F-\bar{F}) / 2}}+\text { h.c. }=\mathcal{L}_{P E}
\end{aligned}
$$

Furthermore

$$
\begin{aligned}
\mathcal{L}_{Y M}^{\prime} & =\frac{1}{8} \int d^{4} \theta \frac{E^{\prime}}{R^{\prime}} f^{\prime} W^{\prime \alpha} W_{\alpha}^{\prime}+\text { h.c. } \\
& =\frac{1}{8} \int d^{4} \theta \frac{E f W^{\alpha} W_{\alpha} e^{-(F-\bar{F}) / 2}}{R e^{-(F-\bar{F}) / 2}}+\text { h.c. }=\mathcal{L}_{Y M}
\end{aligned}
$$

That is, the complete superfield Lagrangian is invariant under the Kähler transformations $\kappa^{2} K \rightarrow \kappa^{2} K+F+\bar{F}$ and (2.12). 


\section{Gauge invariance:}

This follows from the fact that both $E$ and $R$ are functions of the Kähler potential $K$ which, in turn, is invariant under Yang-Mills transformations of the charged superfields $\Phi_{k}$. These are given by

$$
\begin{aligned}
& e^{V} \rightarrow e^{-i \bar{\Lambda}} e^{V} e^{i \Lambda} \\
& \Phi_{k} \rightarrow e^{-i \Lambda} \Phi_{k}
\end{aligned}
$$

where

$$
\overline{\mathcal{D}}^{\dot{\alpha}} \Lambda=0
$$

and $V=V^{(r)} T^{(r)}$ denotes the Yang-Mills prepotential vector superfield (the $T^{(r)}$ denote the hermitian generators of the Yang-Mills gauge group $H$ ). The square of the YangMills superfield fieldstrength, $W^{\alpha} W_{\alpha}$, is by construction invariant under Yang-Mills transformations.

\section{Lorentz invariance:}

This follows from the fact that all the superfield combinations appearing in $\mathcal{L}_{0}$ and $\mathcal{L}_{Y M}$ as well as in $\mathcal{L}_{P E}$ are scalars with respect to Lorentz transformations.

Invariances $(1)$ - (3) of the tree-level theory of the supergravity-matter supermultiplet system can, of course, also be displayed at component level. Component fields are defined according to standard notation [5, 6, 7]: $A^{i}, \chi_{\alpha}^{i}, \mathcal{F}^{i}$ for chiral multiplets (and similar notations for antichiral multiplets) and $\lambda^{\alpha}, v_{m}, \mathcal{D}$ for Yang-Mills multiplets. The irreducible minimal supergravity multiplet is realized by $\left(e_{m}{ }^{a}, \psi_{m}^{\alpha}, M, b_{a}\right) . M$ and $b_{a}$ denote the auxiliary component fields of minimal supergravity. The covariant derivative of a generic Weyl fermion will, in a theory with invariances (1) - (3), then contain a connection for each of these symmetries.

1. The component connection for gauging Kähler transformations is given by the lowest component [5, 6, 7] of the $U_{K}(1)$ gauge connection superfield $A_{\alpha \dot{\alpha}}$

$$
A_{\alpha \dot{\alpha}}\left|=-\frac{i}{8}\left[\mathcal{D}_{\alpha}, \overline{\mathcal{D}}_{\dot{\alpha}}\right] K\right|=a_{\alpha \dot{\alpha}}=\sigma_{\alpha \dot{\alpha}}^{m} a_{m}
$$


where

$$
a_{m}=\frac{1}{4}\left(\partial_{j} K \mathcal{D}_{m} A^{j}-\partial_{\bar{j}} K \mathcal{D}_{m} \bar{A}^{\bar{j}}\right)+i \frac{1}{4} g_{i \bar{j}}\left(\chi^{i} \sigma^{m} \bar{\chi}^{\bar{j}}\right)
$$

Here, $g_{i \bar{j}}$ denotes the Kähler metric $g_{i \bar{j}}=\partial_{i} \partial_{\bar{j}} K$ of the matter manifold parameterized by $A^{i}$ and $\bar{A}^{\bar{j}}$. Under Kähler transformations

$$
a_{m}{ }^{\prime}=a_{m}+\kappa^{-2} \frac{i}{2} \partial_{m} \operatorname{Im} F .
$$

All component Weyl fermions transform under Kähler transformations (2.2). The component matter Weyl fermion, $\chi_{\alpha}^{i}=\left(\frac{1}{\sqrt{2}}\right) \mathcal{D}_{\alpha} \Phi_{i} \mid$, transforms as

$$
\chi^{\prime i}=e^{(i / 2 \operatorname{Im} F)} \chi^{i}
$$

whereas the gaugino $\lambda^{\alpha}$ transforms with opposite charge

$$
\lambda^{\prime \alpha}=e^{(-i / 2 \operatorname{Im} F)} \lambda^{\alpha}
$$

as does the gravitino $\psi_{m}^{\alpha}$.

2. The component Yang-Mills connection $v_{\alpha \dot{\alpha}}=v_{\alpha \dot{\alpha}}^{(r)} T^{(r)}$ is contained in the Yang-Mills prepotential $V$ as

$$
\frac{1}{2}\left[\mathcal{D}_{\alpha}, \overline{\mathcal{D}}_{\dot{\alpha}}\right] V \mid=-2 v_{\alpha \dot{\alpha}}
$$

3. The component Lorentz connection $\omega_{m \alpha}{ }^{\beta}$ is given by the lowest component of the $S L(2, C)$ gauge connection superfield $\phi_{m \alpha}{ }^{\beta}$

$$
\phi_{m \alpha}{ }^{\beta} \mid=\omega_{m \alpha}{ }^{\beta}
$$

Therefore, the covariant derivative for matter fermions $\chi_{\alpha}^{i}$ reads [5, 6, []

$$
\begin{aligned}
\mathcal{D}_{m} \chi^{i} & =\left(\partial_{m}+i v_{m}^{(r)}\left(T^{(r)}-\frac{1}{2} D^{(r)}\right)\right. \\
& \left.-\omega_{m}+\frac{i}{2} b_{m}-\kappa^{2} a_{m} \ldots\right) \chi^{i}
\end{aligned}
$$

where

$$
D^{(r)}=\kappa^{2} \frac{\partial K}{\partial A^{i a}} T_{b}^{(r) a} A^{i b}
$$


The $\ldots$ stand for the additional coupling to the $\sigma$-model Christoffel connection $\Gamma_{j k}^{i}=$ $g^{i j} \partial_{j} g_{j k}$. Such a coupling will not be considered in this paper.

The covariant derivative of the matter scalar $A^{i}$ is given by

$$
\mathcal{D}_{m} A^{i}=\partial_{m} A^{i}+i v_{m}^{(r)} T^{(r)} A^{i}
$$

Note the absence of a coupling of the matter scalar $A^{i}$ to the Kähler connection $a_{m}$ given in (2.19). This is in manifest contrast to the matter fermion $\chi^{i}$. The reason for this is that the Kähler charge $\omega_{K}\left(\Phi_{i}\right)$ of matter superfield $\Phi_{i}$ is zero, $\omega_{K}\left(\Phi_{i}\right)=0$. Therefore, only the component fermions carry Kähler charge and, hence, only component fermions rotate under Kähler transformations (2.2).

We will now expand the supergravity-matter kinetic superfield Lagrangian (2.8) into components. It is, by now, well known that one of the advantages of the Kähler superspace formulation lies in the fact that it immediately gives the correctly normalized kinetic terms for all the component fields without any need for rescalings or complicated partial integrations at the component field level. That is, it immediately gives the correctly normalized Einstein-Hilbert action as well as making the component Kähler structure in the matter sector manifest. The component kinetic Lagrangian for the supergravity-matter system reads [14, [15, 目, 司, 6, 目]

$$
\begin{aligned}
\mathcal{L}_{0} / e & =-\frac{1}{2} \kappa^{-2} e \mathcal{R}-\frac{1}{3} \kappa^{-2}\left(M \bar{M}-b^{a} b_{a}\right) \\
& -g^{m n} g_{i \bar{j}} \mathcal{D}_{n} A^{i} \mathcal{D}_{m} \bar{A}^{\bar{j}}-\frac{i}{2} \chi^{\alpha i} g_{i \bar{j}} \sigma_{\alpha \dot{\alpha}}^{m} \mathcal{D}_{m} \bar{\chi}^{\bar{j} \dot{\alpha}}+\frac{i}{2}\left(\mathcal{D}_{m} \chi^{\alpha i}\right) g_{i \bar{j}} \sigma_{\alpha \dot{\alpha}}^{m} \bar{\chi}^{\bar{j} \dot{\alpha}} \\
& +\mathcal{D}^{(r)} D_{(r)}+\ldots
\end{aligned}
$$

where we have only displayed the component terms relevant for this paper.

Of importance to this paper are the couplings of matter currents to external gravitational fields and to Yang-Mills and Kähler connections. Let us look at the fermionic matter current $g_{i \bar{j}} \chi_{\alpha}^{i} \bar{\chi}_{\dot{\alpha}}^{\bar{j}}$. From the component Lagrangian (2.28) it follows that the fermionic current couples to the Yang-Mills connection $v_{m}^{(r)}$, the Kähler connection $a_{m}$, to the gravitational spin connection $\omega_{m \alpha}{ }^{\beta}$ as well as to the auxiliary field $b_{a}$ and the space-time metric. The bosonic matter current $g_{i \bar{j}} A^{i} \partial_{m} A^{\bar{j}}$, on the other hand, couples only to the Yang-Mills connection $v_{m}^{(r)}$, as well as to the space-time metric $g_{m n}$.

Let us emphasize again how differently both component currents couple to the Kähler connection. The bosonic component current doesn't couple to $a_{m}$ at all! This 
is in manifest contrast to the coupling of both currents to the Yang-Mills connection $v_{m}$. Also note that there is a coupling of $A^{i} \bar{A}^{\bar{j}}$ to the $\mathcal{D}$-term of the Yang-Mills prepotential $V$, through the term $\mathcal{D}^{(r)} D_{(r)}$, whereas there is no such coupling of $A^{i} \bar{A}^{\bar{j}}$ to the highest component of prepotential $K$. The reason for all of this is, once again, obvious. On one hand, the matter superfield $\Phi_{i}$ carries a non-zero Yang-Mills charge, on the other hand it has zero Kähler charge, $\omega_{K}\left(\Phi_{i}\right)=0$. This will be of utmost importance in the next section.

Finally, note that we have not expanded the potential energy (2.9) into component fields, since its component expansion will not be needed in this paper. Its superfield form is, however, relevant to our discussion. The additional matter couplings contained in the Yang-Mills Lagrangian (2.10), on the other hand, are of no relevance for the subsequent discussion of the Kähler anomalous contributions from triangle graphs to the one-particle irreducible effective action. Hence, we will ignore them throughout this paper.

\section{Kähler Anomalies in the Kähler Superspace Formalism}

Since all of the fermions in the supergravity-matter theory are chiral, the possibility exists that all three of the symmetries discussed in the previous section are anomalous at the one-loop level. However, it is well known that there are no pure Lorentz anomalies in four-dimensions. Furthermore, we will assume, as in the standard electroweak model, that the matter superfield content is so chosen that there are no pure gauge anomalies or mixed gauge-Lorentz anomalies. What about possible one-loop anomalies in the Kähler symmetry? It has recently been demonstrated [16, 9, 10, 26] that both non-vanishing pure Kähler, mixed Kähler-gauge and KählerLorentz anomalies, in general, exist. We will not discuss the pure Kähler anomalies in this paper, since their physical relevance is presently obscure. The mixed Kähler anomalies, however, are known to lead to important phenomenological and cosmological effects [16, 10, 26, 11, 12, 25], and it is these mixed anomalies we now analyze in detail.

We begin by considering the mixed Kähler-gauge anomaly. We will, throughout this paper, work to lowest order in supergravity and in Kähler background fields. 
Consider a set of matter chiral superfields, $\Phi_{i}$, a subset of which have non-vanishing gauge charges, so chosen as to satisfy the condition that they are free of pure gauge anomalies. The relevant part of the supergravity-matter Lagrangian is given in (2.28), with the covariant derivatives given by (2.25) and (2.27). The graph in Figure 1a gives the fermionic contribution to the mixed Kähler-gauge anomaly. This graph can be evaluated [1] and the contribution to the associated effective Lagrangian is found to be

$$
\mathcal{L}_{\chi}=\frac{i \kappa^{2}}{(4 \pi)^{2}} \operatorname{Tr} F_{m n} \tilde{F}^{m n} \frac{1}{\square_{0}} \partial^{p} a_{p}
$$

where $F_{m n}$ is the covariant curl of $v_{m}, \tilde{F}^{m n}=\frac{1}{2} \epsilon^{m n \ell p} F_{\ell p}$ and $\square_{0}$ denotes the flat space d'Alembertian. Implied in (3.1) is a sum over all charged chiral matter multiplets $\Phi_{i}$. It can readily be seen that (3.1) is anomalous under Kähler transformations. Using (2.20) we find that

$$
\delta_{K} \mathcal{L}_{\chi}=-\frac{1}{2} \frac{\kappa^{2}}{(4 \pi)^{2}} \operatorname{Im} F \operatorname{Tr} F_{m n} \tilde{F}^{m n}
$$

which, since it doesn't vanish, implies that Kähler symmetry is broken. Now $\mathcal{L}_{\chi}$ by itself is not supersymmetric. Before trying to find the one-loop graphs that will supersymmetrize (3.1), it is worth noting that there is a unique superfield expression whose highest component contains $\mathcal{L}_{\chi}$. This expression is given by

$$
\mathcal{L}=-\frac{1}{8} \frac{\kappa^{2}}{(4 \pi)^{2}} \int d^{4} \theta \operatorname{Tr} W_{\alpha}^{2} \frac{1}{\square_{0}} D^{2} K+\text { h.c. }
$$

Expanding (3.3) out into component fields yields

$$
\begin{aligned}
\mathcal{L} & =\frac{i \kappa^{2}}{(4 \pi)^{2}} \operatorname{Tr} F_{m n} \tilde{F}^{m n} \frac{1}{\square_{0}} \partial^{p} a_{p} \\
& -\frac{\kappa^{2}}{8(4 \pi)^{2}} \operatorname{Tr} F_{m n} F^{m n} \frac{1}{\square_{0}}\left(\left.4 K\right|_{\theta^{2} \bar{\theta}^{2}}+\square_{0} K \mid\right) \\
& +\ldots
\end{aligned}
$$

where ... refers to terms containing fermions. $K \mid$ and $\left.K\right|_{\theta^{2} \bar{\theta}^{2}}$ are the lowest and $\theta^{2} \bar{\theta}^{2}$ components of superfield $K\left(\Phi_{i}, \Phi_{i}^{\dagger}\right)$ respectively given by

$$
\begin{aligned}
K \mid & =K\left(A^{i}, \bar{A}^{\bar{i}}\right) \\
\left.K\right|_{\theta^{2} \bar{\theta}^{2}} & =-g_{i \bar{j}} \mathcal{D}_{m} A^{i} \mathcal{D}^{m} \bar{A}^{\bar{j}}+g_{i \bar{j}} \mathcal{F}^{i} \overline{\mathcal{F}}^{\bar{j}}+\frac{1}{4} \square_{0} K+\ldots
\end{aligned}
$$


where ... stand for terms containing fermions. Now the term in (3.4) proportional to $\operatorname{Tr} F_{m n} F^{m n}$ is $C P$ even and would have to be generated by a one-loop graph with scalar fields running around the loop. But, it is clear from (2.28) that there is no quadratic coupling of $A^{i}$ to either $K \mid$ or $\left.K\right|_{\theta^{2} \bar{\theta}^{2}}$. Therefore, there are no one-loop graphs that can generate the second term in (3.4)! It is, apparently, impossible to construct a supersymmetric expression for the mixed Kähler-gauge anomaly using one-loop graphs generated from the Kähler superspace Lagrangian. There have been some attempts to show that the missing graphs arise from gauge field two-point functions through the regularization procedure. However, these attempts explicitly break gauge invariance when $K\left(A^{i}, \bar{A}^{\bar{i}}\right)$ is non-constant and must, in our opinion, be discarded. Before showing how to perform a supersymmetric calculation, we demonstrate that the same problem exists for the mixed Kähler-Lorentz anomalies.

Again, the relevant part of the supergravity-matter Lagrangian is given in (2.28) with the covariant derivatives given by (2.25) and (2.27). The graph in Figure 1b gives the fermionic contribution to the mixed Kähler-Lorentz anomaly. This graph can be evaluated [1] and the contribution to the associated effective Lagrangian, steming from one Weyl fermion running in the loop, is found to be

$$
\mathcal{L}_{\chi}^{\prime}=-i \frac{\kappa^{2}}{24(4 \pi)^{2}} \mathcal{R}_{m n b}{ }^{a} \tilde{\mathcal{R}}^{m n}{ }_{a}{ }^{b} \frac{1}{\square_{0}} \partial^{p} a_{p}
$$

where $\mathcal{R}_{m n b}{ }^{a}$ is the Lie algebra valued curvature tensor and $\tilde{\mathcal{R}}^{m n}{ }_{a}{ }^{b}=\frac{1}{2} \epsilon^{m n \ell p} \mathcal{R}_{\ell p a}{ }^{b}$. If there are $N$ chiral matter fermions in the theory, then (3.6) would simply be multiplied by $N$. It is easy to see using (2.20) that (3.6) is not invariant under Kähler transformations. Now, $\mathcal{L}_{\chi}^{\prime}$ by itself is not supersymmetric. Before trying to find the one-loop graphs that will supersymmetrize (3.6), it is worth noting that there is a minimal superfield expression whose highest component contains $\mathcal{L}_{\chi}^{\prime}$. This expression is given by

$$
\mathcal{L}^{\prime}=-\frac{\kappa^{2}}{24(4 \pi)^{2}} \int d^{4} \theta W_{\alpha \beta \gamma}^{2} \frac{1}{\square_{0}} D^{2} K+\text { h.c. }
$$

Expanding (3.7) out into component fields [30, 21] yields

$$
\mathcal{L}^{\prime}=-\frac{i \kappa^{2}}{24(4 \pi)^{2}} \mathcal{R}_{m n b}{ }^{a} \tilde{\mathcal{R}}^{m n}{ }_{a}^{b} \frac{1}{\square_{0}} \partial^{p} a_{p}
$$




$$
\begin{aligned}
& +\frac{\kappa^{2}}{192(4 \pi)^{2}} C_{m n p q} C^{m n p q} \frac{1}{\square_{0}}\left(\left.4 K\right|_{\theta^{2} \bar{\theta}^{2}}+\square_{0} K \mid\right) \\
& +\ldots
\end{aligned}
$$

where ... refers to terms containing fermions and $\mathcal{C}_{m n p q}$ denotes the Weyl tensor. Now the term in (3.8) proportional to $C_{m n p q} C^{m n p q}$ is $C P$ even and would have to be generated by a one-loop graph with scalar fields running around the loop. But,again, it is clear from (2.28) that there is no quadratic coupling of $A^{i}$ to either $K \mid$ or $\left.K\right|_{\theta^{2} \bar{\theta}^{2}}$. Therefore, there are no one-loop graphs that can generate the second term in (3.8)! Again, it is impossible to construct a supersymmetric expression for the mixed Kähler-Lorentz anomaly using one-loop graphs generated from the Kähler superspace Lagrangian. Furthermore, any attempt to generate the missing graphs from the graviton two-point function using the regularization procedure will break Lorentz invariance.

As paradoxical as these results might seem, it is not too hard to understand their origin. In superfields, the gauge prepotential couples to matter as $\Phi^{\dagger} e^{V} \Phi$. Were we to examine the pure gauge anomaly three-point function, we would to lowest order in $V$ get as a result the supersymmetric expression

$$
\mathcal{L}^{\prime \prime} \sim \int d^{4} \theta \operatorname{Tr} W_{\alpha}^{2} \frac{1}{\square} D^{2} V+\text { h.c. }
$$

If the Kähler prepotential also coupled to matter as $\Phi^{\dagger} e^{K} \Phi$, then we would indeed have arrived at the supersymmetric expressions (3.3) and (3.7) for the mixed Kähler-gauge and Kähler-Lorentz anomalies. However, in Kähler superspace $\omega_{K}\left(\Phi_{i}\right)=0$ ! It follows that there is no such direct coupling of $K$ to quadratic matter superfields and, hence, one expects difficulty computing a supersymmetric expression for the anomaly. Two things, then, seem clear. First, it must be possible to directly calculate a supersymmetries expression for the mixed Kähler anomalies. Secondly, it seems likely that we must go to a formalism where $K$ couples to matter as $\Phi^{\dagger} e^{K} \Phi$. We show how to do this in the next section.

\section{Conventional Superspace and Tree-Level Symmetries}

As discussed in the introduction, the Lagrangian describing the coupling of $D=4$, $N=1$ supergravity to matter supermultiplets has, in general, a non-canonical gravitational kinetic energy of the form $h(A, \bar{A}) \mathcal{R}$. In the previous sections we showed that 
if we worked with appropriately rescaled fields so that $h(A, \bar{A})=1$, then the tree-level Lagrangian was explicitly invariant under Kähler symmetry. However, computing the one-loop supersymmetric mixed Kähler anomalies with this Lagrangian is apparently impossible, for the reasons discussed above. In this section, we go back to the generic supergravity-matter Lagrangian where $h(A, \bar{A}) \neq 1$ and gravity is not canonically normalized. We will show that, in this case, the Kähler potential couples to quantum matter in the generic form $\Phi^{\dagger} e^{K} \Phi$ and that accordingly pure Kähler symmetry is replaced by a mixed super-Weyl-Kähler symmetry at the tree-level. This, as we will see, is the first step toward a coherent calculation of mixed supersymmetric Kähler anomalies.

In the generic case, the structure group of superspace is taken to be simply $S L(2, C)$ with the associated one-form gauge connection $\phi_{B}{ }^{A}=d z^{M} \phi_{M B}{ }^{A}$. In addition, one introduces the supervielbein $E_{M}{ }^{A}$ and the associated one-forms $E^{A}=d z^{M} E_{M}{ }^{A}$. Solving the Bianchi identities subject to a set of constraints [22, one finds that all components of the torsion and curvature may be expressed in terms of a set of superfields and their coordinate derivatives:

$$
\text { superfield } R, R^{\dagger} \quad G_{\alpha \dot{\alpha}} \quad W_{\alpha \beta \gamma}, \quad \bar{W}_{\dot{\alpha} \dot{\beta} \dot{\gamma}}
$$

Since they are obtained by solving the Bianchi identities with respect to a very different set of constraints, the $R, G_{\alpha \dot{\alpha}}$ and $W_{\alpha \beta \gamma}$ in this section are different than, and not to be confused with, the field strength solutions of the Bianchi identities in Kähler superspace. The relation between them will be discussed in detail later. If we further assume that there is an internal gauge group, then we must introduce yet another oneform gauge connection $\mathcal{A}_{a}{ }^{b}=d z^{M} \mathcal{A}_{M a}{ }^{b}$. Solving the Bianchi identities now introduces a new superfield strength, $W_{\alpha}^{a}$.

Using these superfields, one can write down the tree-level superfield Lagrangian in this superspace. Again, it consists of three parts, each specified by a fundamental function. The first part is the supergravity-matter kinetic energy term given by

$$
\mathcal{L}_{0}=-3 \kappa^{-2} \int d^{4} \theta E e^{-\frac{\kappa^{2}}{3} K\left(\Phi_{i}, \Phi_{i}^{\dagger}\right)}
$$

The second part is the potential energy term given by

$$
\mathcal{L}_{P E}=\frac{1}{2} \int d^{4} \theta \frac{E}{R} W\left(\Phi_{i}\right)+\text { h.c. }
$$


Finally, the Yang-Mills Lagrangian is

$$
\mathcal{L}_{Y M}=\frac{1}{8} \int d^{4} \theta \frac{E}{R} f\left(\Phi_{i}\right)_{a b} W^{\alpha a} W_{\alpha}{ }^{b}+\text { h.c. }
$$

Note that $E$ is the superdeterminant in conventional $S L(2, C)$ superspace and is not identical to the superdeterminant in Kähler superspace discussed earlier. Now, the total tree-level Lagrangian possesses, by construction, both gauge and Lorentz invariance, as did the Kähler superspace Lagrangian. However, it is clear that under the Kähler transformation $\kappa^{2} K \rightarrow \kappa^{2} K+F+\bar{F}$ (4.2), for example, is not invariant. That is, the conventional supergravity-matter Lagrangian does not possess pure Kähler symmetry. Is there anything that replaces it? After a short discussion, we will show that indeed there is, as is well known 14, 15, 31.

The conventional torsion constraints are left invariant [24] under a set of superscaling transformations called super-Weyl or Howe-Tucker transformations. They change the forms of superspace as follows

$$
\begin{aligned}
& E_{M}{ }^{a} \rightarrow e^{\Sigma+\bar{\Sigma}} E_{M}{ }^{a} \\
& E_{M}{ }^{\alpha} \rightarrow e^{2 \bar{\Sigma}-\Sigma}\left(E_{M}{ }^{\alpha}+\frac{i}{2} E_{M}{ }^{b}\left(\epsilon \sigma_{b}\right)^{\alpha}{ }_{\dot{\alpha}} \overline{\mathcal{D}}^{\dot{\alpha}} \bar{\Sigma}\right) \\
& E_{M \dot{\alpha}} \rightarrow e^{2 \Sigma-\bar{\Sigma}}\left(E_{M \dot{\alpha}}+\frac{i}{2} E_{M}{ }^{b}\left(\epsilon \bar{\sigma}_{b}\right)_{\dot{\alpha}}{ }^{\alpha} \mathcal{D}_{\alpha} \Sigma\right)
\end{aligned}
$$

where $\Sigma$ and $\bar{\Sigma}$ are superfield parameters subject to the chirality conditions

$$
\begin{aligned}
& \overline{\mathcal{D}}^{\dot{\alpha}} \Sigma=0 \\
& \mathcal{D}_{\alpha} \bar{\Sigma}=0
\end{aligned}
$$

It is not hard to show that under (4.5)

$$
E \rightarrow E e^{2(\Sigma+\bar{\Sigma})}
$$

Chiral superfields and $K\left(\Phi_{i}, \Phi_{i}^{\dagger}\right)$ are invariant under super-Weyl transformations. It is clear that (4.2), for example, and, hence, the conventional supergravity-matter Lagrangian is not invariant under pure super-Weyl rescalings. However, under combined Kähler and super-Weyl transformations (4.2) transforms as

$$
\begin{aligned}
\mathcal{L}_{0}^{\prime} & =-3 \kappa^{-2} \int d^{4} \theta E^{\prime} e^{-\frac{\kappa^{2}}{3} K^{\prime}} \\
& =-3 \kappa^{-2} \int d^{4} \theta E e^{2(\Sigma+\bar{\Sigma})} e^{-\frac{\kappa^{2}}{3} K^{\prime}} e^{-\frac{1}{3}(F+\bar{F})}
\end{aligned}
$$


It is clear that if we take

$$
\begin{aligned}
\Sigma & =\frac{1}{6} F \\
\bar{\Sigma} & =\frac{1}{6} \bar{F}
\end{aligned}
$$

then Lagrangian (4.2) will be invariant. Similarly, if one demands that under Kähler transformations, the superpotential transform as

$$
W \rightarrow e^{-F} W
$$

then, using the fact that under super-Weyl rescalings

$$
\begin{aligned}
\delta R & =-2(2 \Sigma-\bar{\Sigma}) R-\frac{1}{4} \overline{\mathcal{D}}^{2} \bar{\Sigma} \\
W_{\alpha} & \rightarrow e^{-3 \Sigma} W_{\alpha}
\end{aligned}
$$

it follows that $\mathcal{L}_{P E}$, (4.3), and $\mathcal{L}_{Y M}$, (4.4), will also be invariant under combined superWeyl-Kähler transformations with $\Sigma$ given in (4.9). We conclude, therefore that instead of possessing pure Kähler symmetry, the conventional supergravity-matter tree-level Lagrangian exhibits a mixed super-Weyl-Kähler invariance [14, [15, 31].

Before considering the component field Lagrangian, it is very helpful to first reexpress $\mathcal{L}_{0}$ in (4.2) and $\mathcal{L}_{P E}$ in (4.3) in a way that exposes, at the superfield level, the vertices relevant to the anomaly calculation. As in Kähler superspace, $\mathcal{L}_{Y M}$ in (4.4) does not contribute to Kähler anomalous one-loop irreducible graphs and will, henceforth, be ignored. To exhibit the relevant vertices, we perform a background fieldquantum field splitting of the chiral matter superfields as well as of the supergravity superfields in such a way that, all relevant quantum superfields are invariant under super-Weyl-Kähler transformations of the background superfields. This proceeds as follows. First perform a background field-quantum field splitting of the chiral matter superfields as

$$
\Phi_{i}=\Phi_{c i}+\phi_{i}
$$

where $\Phi_{c i}$ is a background superfield and $\phi_{i}$ is the fluctuating quantum superfield. Henceforth, for simplicity of notation, we will suppress the index $i$. Recalling that $\Phi$ does not transform under super-Weyl-Kähler transformations, and demanding that $\Phi_{c}$ 
also not transform, it follows that under super-Weyl-Kähler transformations

$$
\delta \phi=0
$$

To proceed further, we must discuss the background field-quantum field splitting of the supergravity superfields. It is well known [20] that the superdeterminant $E$ can be written in terms of a set of supergravity prepotentials $H^{A}$ and $\rho$, where $\rho$ is a chiral superfield. In terms of these prepotentials $E$ factors into

$$
E=\hat{E}\left[H^{A}\right] e^{\rho+\bar{\rho}}
$$

where $\hat{E}$ is a complicated function of $H^{A}$ whose exact form is irrelevant for this paper. What is relevant is that under super-Weyl transformations $\hat{E}$ is invariant. Superprepotential $\rho$, on the other hand, transform as

$$
\rho \rightarrow \rho+2 \Sigma
$$

under super-Weyl transformations, which correctly reproduces (4.7). Recall that under super-Weyl-Kähler transformations $\Sigma$ satisfies (4.9) and, therefore, that

$$
\rho \rightarrow \rho+\frac{1}{3} F
$$

We now perform a background field-quantum field splitting of $\rho$ as

$$
\rho=\rho_{c}+\rho_{q}
$$

where $\rho_{c}$ is a chiral background superfield and $\rho_{q}$ a fluctuating chiral quantum superfield. Using (4.16), and demanding that

$$
\rho_{c} \rightarrow \rho_{c}+\frac{1}{3} F_{c}
$$

it follows from (4.17) that under super-Weyl-Kähler transformations

$$
\rho_{q} \rightarrow \rho_{q}+\frac{1}{3} F_{\phi c} \phi+\frac{1}{6} F_{\phi \phi c} \phi^{2}+\cdots
$$

where the subscript $\phi$ means differentiation with respect to these fields and subscript $c$ means evaluation at the classical background superfield. Note that this transformation mixes quantum gravity superfields with quantum matter superfields. Amongst 
other things, this implies that the relevant part of the naive path integral measure, $[\mathcal{D} \phi]\left[\mathcal{D} \phi^{\dagger}\right]\left[\mathcal{D} \rho_{q}\right]\left[\mathcal{D} \rho_{q}^{\dagger}\right]$, is not invariant under super-Weyl-Kähler transformations. This can be cured by introducing the appropriate Jacobian determinant but there is an easier approach, which we will now follow. This consists of defining a new chiral quantum supergravity superfield $\rho_{q}^{\prime}$ by

$$
\rho_{q}^{\prime}=\rho_{q}+\frac{1}{3}(\ln W)_{\phi c} \phi+\frac{1}{6}(\ln W)_{\phi \phi c} \phi^{2}+\cdots
$$

Combining (4.10), (4.19) and (4.20), it follows that under super-Weyl-Kähler transformations

$$
\delta \rho_{q}^{\prime}=0
$$

In this new variable, the relevant part of the path integral measure, $[\mathcal{D} \phi]\left[\mathcal{D} \phi^{\dagger}\right]\left[\mathcal{D} \rho_{q}^{\prime}\right]\left[\mathcal{D} \rho_{q}^{\prime \dagger}\right]$ with unit Jacobian, is indeed invariant under super-Weyl-Kähler transformations. Henceforth, we will use $\phi$ and $\rho_{q}^{\prime}$ as the fluctuating quantum superfields.

We will first display the couplings of uncharged quantum matter superfields $\phi$ to background supergravity and background matter fields. Expanding $K\left(\Phi, \Phi^{\dagger}\right)$ to quadratic order around classical background $\Phi_{c}$ gives

$$
\begin{aligned}
K= & K_{c}+K_{\phi c} \phi+K_{\phi^{\dagger} c} \phi^{\dagger} \\
& +K_{\phi \phi^{\dagger} c} \phi^{\dagger} \phi \\
& +\frac{1}{2} K_{\phi \phi c} \phi^{2}+\frac{1}{2} K_{\phi^{\dagger} \phi^{\dagger} c} \phi^{\dagger^{2}}+\ldots
\end{aligned}
$$

where, again, the subscript $\phi$ or $\phi^{\dagger}$ means differentiation with respect to these fields and subscript $c$ means evaluation at the classical background superfields. A similar expansion holds for the holomorphic superpotential $W$. Substitute these expansions into Lagrangians (4.2) and (4.3) and use (4.14), (4.17) and (4.20) to express the superdeterminant. Setting the terms linear in $\phi$ to zero leads to the following equation of motion for background field $\Phi_{c}$

$$
\left(\overline{\mathcal{D}}^{2}-8 R\right)_{c}\left[e^{-\frac{\kappa^{2}}{3} K_{c}} G_{\phi c}\right]=0
$$

where $G$ denotes the Kähler invariant combination $G=K+\ln W+\ln \bar{W}$. Note that the equation of motion (4.23) is manifestly covariant under super-Weyl-Kähler transformations of the background superfields. Also note that we have ignored a possible 
contribution of Lagrangian (4.4) to the equation of motion for $\Phi_{c},(4.23)$. As stated earlier, such matter couplings do not lead to super-Weyl-Kähler anomalous contributions steming from irreducible graphs. The resulting Lagrangian now becomes

$$
\begin{aligned}
\mathcal{L}_{0}+\mathcal{L}_{P E}= & -3 \kappa^{-2} \int d^{4} \theta E_{c} e^{-\frac{\kappa^{2}}{3} K_{c}}+\left(\frac{1}{2} \int d^{4} \theta \frac{E_{c}}{R_{c}} W_{c}+\text { h.c. }\right) \\
& +\int d^{4} \theta E_{c} e^{-\frac{\kappa^{2}}{3} K_{c}}\left[\left(g_{\phi \phi^{\dagger}}-\frac{\kappa^{2}}{3} G_{\phi} G_{\phi^{\dagger}}\right)_{c} \phi^{\dagger} \phi\right. \\
& +\frac{1}{2}\left(G_{\phi \phi}-\frac{\kappa^{2}}{3} G_{\phi} G_{\phi}\right)_{c} \phi^{2} \\
& \left.+\frac{1}{2}\left(G_{\phi^{\dagger} \phi^{\dagger}}-\frac{\kappa^{2}}{3} G_{\phi^{\dagger}} G_{\phi^{\dagger}}\right)_{c} \phi^{\dagger^{2}}\right]+\ldots
\end{aligned}
$$

where we have dropped all terms depending on $\rho_{q}^{\prime}$, since we will not compute gravitational radiative corrections in this paper. For the same reason we have simply replaced $H^{A}$ by $H_{c}^{A}$ in $\hat{E}$. Lagrangian (4.24) displays the couplings of uncharged quantum superfields $\phi$ to supergravity and background matter fields. We now proceed to display the couplings of charged quantum superfields $\phi$. We will throughout the paper assume that all chiral matter superfields that carry non-vanishing charge under some YangMills gauge group have a vanishing background. Then, the corresponding Lagrangian reads

$$
\begin{aligned}
\mathcal{L}_{0}= & -3 \kappa^{-2} \int d^{4} \theta E_{c} e^{-\frac{\kappa^{2}}{3} K_{c}} \\
& +\int d^{4} \theta E_{c} e^{-\frac{\kappa^{2}}{3} K_{c}} g_{\phi \phi^{\dagger} c} \phi^{\dagger} e^{V} \phi+\ldots
\end{aligned}
$$

Note that terms proportional to $\phi^{2}$ and to $\phi^{\dagger^{2}}$ do not appear. Both Lagrangians (4.24) and (4.25) are manifestly invariant under super-Weyl-Kähler transformations (4.9) of the background superfields. Also note that the terms in (4.24) proportional to $\phi^{2}$ and $\phi^{\dagger^{2}}$ come multiplied by the super-Weyl-Kähler invariant, but background field dependent metrics $G_{\phi \phi c}-\frac{\kappa^{2}}{3} G_{\phi c} G_{\phi c}$ and $G_{\phi^{\dagger} \phi^{\dagger} c}-\frac{\kappa^{2}}{3} G_{\phi^{\dagger c}} G_{\phi^{\dagger} c}$, respectively.

We are now ready to expand Lagrangians (4.24) and (4.25) into component fields using the standard techniques. The relevant part of the Lagrangian given in (4.24) reads, to lowest order in the background fields,

$$
\mathcal{L}_{0} / e=\ldots+\left(g_{A A^{\dagger}}-\frac{\kappa^{2}}{3} G_{A} G_{A^{\dagger}}\right)_{c} e^{-\frac{\kappa^{2}}{3} K_{c} \mid}
$$




$$
\begin{aligned}
& {\left[\left\{\frac{1}{6} \bar{A} A\left(\mathcal{R}-\frac{\kappa^{2}}{2}\left(\left.4 K\right|_{\theta^{2} \bar{\theta}^{2}}+\square_{0} K \mid\right)\right)_{c}\right.\right.} \\
& -g^{m n} \tilde{\mathcal{D}}_{m} A \tilde{\mathcal{D}}_{n} \bar{A} \\
& \left.-\frac{i}{2}\left(\chi \sigma^{m} \tilde{\mathcal{D}}_{m} \bar{\chi}-\left(\tilde{\mathcal{D}}_{m} \chi\right) \sigma^{m} \bar{\chi}\right)\right\} \\
& +\left\{\frac { ( G _ { A A } - \frac { \kappa ^ { 2 } } { 3 } G _ { A } G _ { A } ) _ { c } } { ( g _ { A A ^ { \dagger } } - \frac { \kappa ^ { 2 } } { 3 } G _ { A } G _ { A ^ { \dagger } } ) _ { c } } \left[\frac { 1 } { 1 2 } \left(\mathcal{R}-\frac{\kappa^{2}}{2}\left(\left.4 K\right|_{\theta^{2} \bar{\theta}^{2}}\right.\right.\right.\right. \\
& \left.\left.\left.\left.\left.+\square_{0} K \mid\right)\right)+\frac{1}{6} i \partial^{m} b_{m}\right]_{c} A^{2}+\text { h.c. }\right\}\right] \\
& +\ldots
\end{aligned}
$$

where the covariant derivatives are

$$
\tilde{\mathcal{D}}_{m} A^{i}=\left(\partial_{m}-\frac{i}{3}\left(b_{m}-i 2 \kappa^{2} a_{m}\right)\right) A^{i}
$$

and

$$
\tilde{\mathcal{D}}_{m} \chi^{i}=\left(\partial_{m}-\omega_{m}-\frac{2}{3} \kappa^{2} c_{m}\right) \chi^{i}
$$

and where

$$
c_{m}=a_{m}-\kappa^{-2} \frac{i}{4} b_{m}
$$

Note that $b_{m}, a_{m}, \omega_{m}$ and $c_{m}$ are all evaluated at classical background field values. We omit the subscript $c$ to prevent a confusing proliferation of notation. The appearance of the connections $b_{m}$ and $a_{m}$ in (4.26) and, in particular, the appearance of the exact combination $c_{m}$ requires some explanation. In the conventional superspace $\chi^{i}$ does not transform under pure Kähler transformations. However, under super-Weyl transformations $\chi^{i}$ transforms as

$$
\delta \chi^{i}=-(2 \bar{\Sigma}-\Sigma) \mid \chi^{i}
$$

Similarly, under super-Weyl transformations it can be shown that

$$
\delta b_{m}=-3 i \partial_{m}(\bar{\Sigma}-\Sigma) \mid
$$

Note that this variation of $b_{m}$ does not compensate the variation of $\chi^{i}$. It need not, since the Lagrangian is not purely super-Weyl invariant. If we now also perform a 
Kähler transformation, $a_{m}$ transforms as in (2.20). However, if we further demand that (4.9) hold, we then find from (2.20) and (4.31) that

$$
\delta c_{m}=\kappa^{-2} \frac{3}{8} \partial_{m}(F-\bar{F})
$$

which exactly compensates (4.30), as it must. That is, the exact combination $c_{m}=$ $a_{m}-\kappa^{-2} \frac{i}{4} b_{m}$ appearing in the covariant derivative (4.28) acts as a connection which insures invariance under mixed super-Weyl-Kähler transformations. Also note that the combination $b_{m}-2 i \kappa^{2} a_{m}$ appearing in the covariant derivative $\tilde{\mathcal{D}}_{m} A$ in (4.27) is invariant under mixed super-Weyl-Kähler transformations, as it must, since the component scalar field $A$ does not transform under mixed super-Weyl-Kähler transformations.

The component expansion of Lagrangian (4.25) reads, to lowest order in the background fields,

$$
\begin{aligned}
\mathcal{L}_{0} / e= & \ldots g_{A A^{\dagger} c} e^{-\frac{\kappa^{2}}{3} K_{c} \mid}\left\{\frac{1}{6} \bar{A} A\left(\mathcal{R}-\frac{\kappa^{2}}{2}\left(\left.4 K\right|_{\theta^{2} \bar{\theta}^{2}}+\square_{0} K \mid\right)\right)_{c}\right. \\
& -g^{m m} \tilde{\mathcal{D}}_{m} A \mathcal{D}_{n} \bar{A}+\bar{A} T^{(r)} A \mathcal{D}^{(r)} \\
& \left.-\frac{i}{2}\left(\chi \sigma^{m} \tilde{\mathcal{D}}_{m} \bar{\chi}-\left(\tilde{\mathcal{D}}_{m} \chi\right) \sigma^{m} \bar{\chi}\right)\right\} \\
& +\ldots
\end{aligned}
$$

where the covariant derivatives are

$$
\begin{aligned}
& \tilde{\mathcal{D}}_{m} A^{i}=\left(\partial_{m}+i v_{m}^{(r)} T^{(r)}-\frac{i}{3}\left(b_{m}-2 i \kappa^{2} a_{m}\right)\right) A^{i} \\
& \tilde{\mathcal{D}}_{m} \chi^{i}=\left(\partial_{m}-\omega_{m}+i v_{m}^{(r)} T^{(r)}-\frac{2}{3} \kappa^{2} c_{m}\right) \chi^{i}
\end{aligned}
$$

Again, $v_{m}^{(r)}, b_{m}, a_{m}, \omega_{m}$ and $c_{m}$ are to be evaluated at classical background field values. Note that many terms appearing in (4.26) do not appear in (4.33) since we have set charged background fields to zero.

\section{Super-Weyl-Kähler Anomalies in Conventional Superspace - The Yang- Mills Case}

We are now poised to compute mixed super-Weyl-Kähler-gauge and Lorentz anomalies, in exact analogy with the mixed Kähler-gauge and Lorentz anomalies discussed in section 3. A key difference in these calculations is that now the anomalous 
fermionic current couples to connection $c_{m}$ rather than to $a_{m}$. We will, in this section, consider the mixed super-Weyl-Kähler-gauge anomaly only. The mixed gravitational anomalies will be discussed in the next section. We will work to lowest order in the supergravity-Kähler background fields. As before, we consider a set of matter chiral superfields, $\Phi_{i}$, the subset of which with non-vanishing gauge charges so chosen that they are free of gauge and Lorentz anomalies. The relevant part of the supergravity-matter Lagrangian is given in (4.33), with the covariant derivatives given in (4.34). The graph in Figure 2a gives the fermionic contribution to the mixed super-Weyl-Kähler-gauge anomaly. This graph can be evaluated [1] and the associated effective Lagrangian is found to be

$$
\mathcal{L}_{\chi}=\frac{2 i}{3} \frac{\kappa^{2}}{(4 \pi)^{2}} \operatorname{Tr} F_{m n} \tilde{F}^{m n} \frac{1}{\square_{0}} \partial^{p} c_{p} .
$$

In this expression, and in all results for the remainder of this paper, all fields are to be evaluated at their classical background values. We omit the subscript $c$ to avoid a confusing proliferation of notation. Implied in (5.1), as everywhere else in this section, is a sum over all charged matter multiplets $\phi_{i}$. It is readily seen that (5.1) is anomalous under super-Weyl-Kähler transformations. Using (4.32), we find that

$$
\delta_{S W-K} \mathcal{L}_{\chi}=-\frac{1}{2} \frac{1}{(4 \pi)^{2}} \operatorname{Im} F \operatorname{Tr} F_{m n} \tilde{F}^{m n}
$$

which, since it does not vanish, implies that the super-Weyl-Kähler symmetry is broken. Now $\mathcal{L}_{\chi}$ by itself is not supersymmetric. Before trying to find the one-loop graphs that will supersymmetrize (5.1), it is worth noting that there is a unique superfield expression whose highest component contains $\mathcal{L}_{\chi}$. This expression is given by

$$
\mathcal{L}=\frac{1}{4(4 \pi)^{2}} \int d^{2} \theta \operatorname{Tr} W^{\alpha} W_{\alpha} \frac{1}{\square_{0}}\left(4 R^{\dagger}-\frac{\kappa^{2}}{3} D^{2} K\right)+\text { h.c. }
$$

Expanding (5.3) out into component fields yields

$$
\begin{aligned}
\mathcal{L}=\frac{2 i}{3} & \frac{\kappa^{2}}{(4 \pi)^{2}} \operatorname{Tr} F_{m n} \tilde{F}^{m n} \frac{1}{\square_{0}} \partial^{p} c_{p} \\
& -\frac{1}{12} \frac{\kappa^{2}}{(4 \pi)^{2}} \operatorname{Tr} F_{m n} F^{m n} \frac{1}{\square_{0}}\left(\left.4 K\right|_{\theta^{2} \bar{\theta}^{2}}+\square K \mid\right) \\
& -\frac{1}{12(4 \pi)^{2}} \operatorname{Tr} F_{m n} F^{m n} \frac{1}{\square_{0}} \mathcal{R}+\ldots
\end{aligned}
$$


where ... refers to terms containing fermionic and $\left.K\right|_{\theta^{2} \bar{\theta}^{2}}$ and $K \mid$ are given in (3.5). Can one find graphs that will produce the second and third terms on the right hand side of (5.4)? Unlike the case in Kähler superspace, the answer now is affirmative. To begin with, we consider the graph shown in Figure $2 \mathrm{~b}$ where the scalar fields $A^{i}$ run around the loop. The top vertex originates through the quadratic coupling of $A^{i}$ to $\left.4 K\right|_{\theta^{2} \bar{\theta}^{2}}+\square_{0} K \mid$ in the component field Lagrangian (4.33). This is precisely the coupling that was conspicuously absent in Kähler superspace. Evaluation of this graph leads to the following additional contribution to the effective action

$$
\mathcal{L}_{A}=-\frac{\kappa^{2}}{12(4 \pi)^{2}} \operatorname{Tr} F_{m n} F^{m n} \frac{1}{\square_{0}}\left(\left.4 K\right|_{\theta^{2} \bar{\theta}^{2}}+\square K \mid\right)
$$

precisely the second term on the right-hand side of (5.4). The origin and meaning of the third term in (5.4) is a bit more subtle. The relevant graphs are shown in Figure 2c. The top vertices originate through the fermion and scalar kinetic energy terms in (4.33). Evaluation of these graphs leads to the following contribution to the effective action

$$
\mathcal{L}_{\chi, A}=-\frac{1}{3(4 \pi)^{2}} \operatorname{Tr} F_{m n} F^{m n} \frac{1}{\square_{0}}\left(\frac{1}{4} \mathcal{R}\right) .
$$

This is exactly the third term on the right-hand side of (5.4). There are two issues that must be addressed as regards this term. First of all, one might wonder why we did not consider such graphs in our discussion of Kähler superspace. The reason is that in Kähler superspace, $\mathcal{R}$ does not transform under Kähler transformations and, hence, these graphs are irrelevant for the discussion of the Kähler anomaly. In the conventional superspace of this section, however, $\mathcal{R}$ does transform under the combined super-WeylKähler transformations and, therefore, these graphs are relevant. Secondly, we want to point out that the graphs in Figure $2 \mathrm{c}$ are closely related to the gauge two-point function and the associated trace anomaly [19, 17, 29. The gauge two-point function is computed from the graphs in Figure 3. Prior to renormalization these graphs are evaluated to be

$$
\mathcal{L}_{2}=-\frac{1}{(4 \pi)^{2}} \frac{1}{4}\left(\frac{1}{\epsilon}-\ln \frac{\square_{0}}{2 \pi \mu^{2}}\right) \operatorname{Tr} F_{m n} F^{m n}
$$

using dimensional regularization. The energy momentum tensor is defined by

$$
T_{m n}=\frac{2}{\sqrt{-g}} \frac{\delta S}{\delta g^{m n}}
$$


Varying the action associated with (5.7), one finds that the trace of $T_{m n}$ is finite in four-dimensions and given by

$$
T_{m}^{m}=-\frac{1}{2(4 \pi)^{2}} \operatorname{Tr} F_{m n} F^{m n} .
$$

This result occurs because the variation of $\sqrt{-g} \operatorname{Tr} F_{m n} F^{m n}$ in $4-2 \epsilon$ dimensions is non-vanishing and proportional to $\epsilon$. This multiplies the $\frac{1}{\epsilon}$ pole and yields the finite result in (5.9). Now, this is true for the non-renormalized theory. However, when (5.7) is renormalized, the pole $1 / \epsilon$ is removed, and it is clear that the renormalized $T^{m}{ }_{m}=0$ in four-dimensions when evaluated from the graph in Figure 3. Now add graphs 2c to the renormalized gauge two-point effective Lagrangian. The result in four-dimensions is

$$
\mathcal{L}_{2}+\mathcal{L}_{\chi, A}=\frac{1}{4(4 \pi)^{2}} \ln \left[\frac{\square_{0}}{2 \pi \mu^{2}}\left(1-\frac{1}{3 \square_{0}} \mathcal{R}\right)\right] \operatorname{Tr} F_{m n} F^{m n}
$$

It follows from (5.8) that the associated trace of the energy momentum tensor is

$$
T^{m}{ }_{m}=\frac{1}{2(4 \pi)^{2}} \operatorname{Tr} F_{m n} F^{m n}
$$

which is, up to the sign, the same as in (5.9). The right-hand side of (5.11) is the correct expression for the one-loop trace anomaly [29] of gauge indexed chiral multiplets, each of which has two real scalars and one Weyl fermion. Note that it arises entirely from varying $g_{m n}$ in the three-point effective Lagrangian (5.6) and not in the renormalized two-point Lagrangian. Hence, $\mathcal{L}_{\chi A}$ is the term in the renormalized effective Lagrangian that gives rise to the one-loop trace anomaly.

We conclude, then, that the one-loop three-point graphs shown in Figure 2a, b, and c give rise to the supersymmetric effective Lagrangian (5.4) and, equivalently, to the superfield expression for the Lagrangian (5.3). Under super-Weyl-Kähler transformations

$$
\begin{aligned}
\kappa^{2} \delta K & =F+\bar{F} \\
\delta R^{\dagger} & =-\frac{1}{24} D^{2} F
\end{aligned}
$$

it follows that

$$
\delta \mathcal{L}=\frac{1}{2(4 \pi)^{2}} \int d^{2} \theta \operatorname{Tr} W^{\alpha} W_{\alpha} F+\text { h.c. }
$$


which is non-vanishing. Hence, expression (5.3) represents the supersymmetric mixed super-Weyl-Kähler-gauge anomalous term in the effective action. We point out that expression (5.3) could also have been obtained by direct three-point supergraph calculations using the gauge and supergravitational prepotential formalism [20, 9]. We close this section by showing how to transform the anomalous Lagrangian (5.3), obtained in the conventional superspace formulation, over into a result in the Kähler superspace formulation. Both formulations of supergravity-matter are related by particular superfield rescalings of the underlying torsion constraints [7]. This implies, among other things, that the superfields $R$ and $G_{\alpha \dot{\alpha}}$ of the conventional superspace formulation are related to the superfields $R$ and $G_{\alpha \dot{\alpha}}$ of the Kähler superspace formulation by [7]

$$
\begin{aligned}
R \rightarrow R & -\frac{\kappa^{2}}{24} \bar{D}^{2} K \\
G_{\alpha \dot{\alpha}} \rightarrow G_{\alpha \dot{\alpha}} & -\frac{\kappa^{2}}{12}\left[D_{\alpha}, \bar{D}_{\dot{\alpha}}\right] K
\end{aligned}
$$

where we work to linearised level in $K$ only. It follows from (5.14) that

$$
b_{m} \rightarrow b_{m}+2 i \kappa^{2} a_{m}
$$

Applying (5.14) to the anomalous Lagrangian (5.3) yields

$$
\begin{aligned}
\mathcal{L}= & \frac{1}{(4 \pi)^{2}} \int d^{4} \theta \operatorname{Tr} W^{\alpha} W_{\alpha} \frac{1}{\square_{0}} R^{\dagger} \\
& -\frac{1}{8} \frac{\kappa^{2}}{(4 \pi)^{2}} \int d^{4} \theta \operatorname{Tr} W^{\alpha} W_{\alpha} \frac{1}{\square_{0}} D^{2} K+\text { h.c. }
\end{aligned}
$$

Note that Lagrangian (5.16) is given in terms of Kähler superspace superfields $W^{\alpha}, R^{\dagger}$, $K_{c}$. In Kähler superspace the superfield $R^{\dagger}$ transforms, according to (2.6), homogeneously with Kähler charge $\omega_{K}=-2$ under Kähler transformations. It follows that the term proportional to $R^{\dagger}$ in (5.16) is invariant under Kähler transformations. Hence, it can be discarded and the Kähler anomaly is entirely given by the second term in (5.16). That is

$$
\mathcal{L}^{\text {anom }}=-\frac{1}{8} \frac{\kappa^{2}}{(4 \pi)^{2}} \int d^{4} \theta \operatorname{Tr} W^{\alpha} W_{\alpha} \frac{1}{\square_{0}} D^{2} K+\text { h.c. }
$$

Comparing this expression against Lagrangian (3.3) shows that both agree! Hence, it follows that a consistent way of generating supersymmetric one-loop results in the 
Kähler superspace formulation is to first perform manifestly supersymmetric calculations in the conventional formulation, and then to transform them over into the Kähler superspace formulation by using the superfield rescalings of the torsion constraints given in [7]. We will, in the next section, apply this strategy to the computation of the mixed supersymmetric Kähler-Lorentz anomaly in Kähler superspace.

Finally, recall that $\operatorname{Tr} T^{(r)} T^{(s)}=\sum_{H} g_{H}^{2} \sum_{R} c_{R}^{H} \delta^{(r)(s)}$, where each $H$ is a factor group of the total gauge group, $g_{H}$ is the gauge coupling parameter associated with $H$ and $R$ sums over various representations of multiplets within each factor gauge group. It follows that (5.17) can be written as

$$
\mathcal{L}=-\frac{1}{8} \frac{\kappa^{2}}{(4 \pi)^{2}} \sum_{H} g_{H}^{2} \int d^{4} \theta\left(W_{\alpha}^{H(r)}\right)^{2} \frac{1}{\square_{0}} D^{2}\left[\sum_{R} c_{R}^{H} K\right]+\text { h.c. }
$$

In this paper, we have, for simplicity, not discussed Kähler anomalies with internal vector supermultiplet loops. Similarly, we have ignored anomalies involving the sigmamodel Christoffel connection [27, 26, 16, 10]. It seems clear that these calculations will proceed in a manner similar to that discussed above. Assuming this to be the case, the result (5.18) can be easily extended to give the complete supersymmetric Kähler and sigma-model anomalies for both chiral and vector superfield internal loops. The final result is 26, 16, 10,

$$
\mathcal{L}=\frac{1}{8} \frac{\kappa^{2}}{(4 \pi)^{2}} \sum_{H} g_{H}^{2} \int d^{4} \theta\left(W_{\alpha}^{H(r)}\right)^{2} \frac{1}{\square_{0}} D^{2}\left[c_{V}^{H} K+\sum_{R} c_{R}^{H}\left(2 \operatorname{lndet} g_{R}^{H}-K\right)\right]+\text { h.c. }
$$

\section{Super-Weyl-Kähler Anomalies in Conventional Superspace - The Grav- itational Case}

We now turn to the computation of the mixed super-Weyl-Kähler-Lorentz anomaly. We will, again, work to lowest order in supergravity-Kähler background fields. All results presented in this section are for one chiral matter supermultiplet running in the loop. The generalization of these results to $N$ chiral quantum matter supermultiplets is obtained by multiplying all the results of this section by $N$. The relevant part of the supergravity-matter Lagrangian is given in (4.26) with the covariant derivatives given in (4.27) and (4.28). The graph in Figure 4a gives the fermionic contribution to the mixed super-Weyl-Kähler-Lorentz anomaly. This graph can be evaluated [1] and the 
associated effective Lagrangian is found to be

$$
\mathcal{L}_{\chi}^{\prime}=-\frac{2 i \kappa^{2}}{3} \frac{1}{24} \frac{1}{(4 \pi)^{2}} \mathcal{R}_{m n a}{ }^{b} \tilde{\mathcal{R}}^{m n a}{ }_{b} \frac{1}{\square_{0}} \partial^{p} c_{p} .
$$

Proceeding by analogy with the previous case of the mixed gauge anomaly, we now also evaluate three-point graphs with scalar fields $A, \bar{A}$ running in the loop. Unlike the previous case, however, there are now $A^{2}$ and $\bar{A}^{2}$ as well as $\bar{A} A$ vertices to consider. These vertices fall into two categories, the "conformal" vertices $A \bar{A}$ and the "conformal breaking" vertices $A^{2}$ and $\bar{A}^{2}$. The conformal vertices arise from the $\phi^{\dagger} \phi$ term in Lagrangian (4.24), which is invariant under the conformal transformations. These are defined by arbitrary super-Weyl transformations, as given in (4.7), accompanied by the superfield rescalings of the quantum fields $\phi$ and $\phi^{\dagger}$, given by

$$
\begin{aligned}
\phi & \rightarrow e^{-2 \Sigma} \phi \\
\phi^{\dagger} & \rightarrow e^{-2 \bar{\Sigma}} \phi^{\dagger} .
\end{aligned}
$$

From (6.2) it follows immediately that the $\phi^{2}$ and $\phi^{\dagger^{2}}$ terms in Lagrangian (4.24) are not invariant under the conformal transformations. Hence, the $A^{2}$ and $\bar{A}^{2}$ vertices in the component Lagrangian (4.26) are of the conformal breaking type.

We begin by evaluating three-point graphs constructed from the conformal vertices only. These graphs are shown in Figures $4 \mathrm{~b}$ and 4c. The graph in Figure $4 \mathrm{~b}$ has scalars running around the loop. We find that its contribution to the effective Lagrangian is

$$
\begin{aligned}
& \mathcal{L}_{A}^{\prime}=\frac{\kappa^{2}}{12 \cdot 24 \cdot(4 \pi)^{2}}\left(d_{M} C_{m n p q} C^{m n p q}-\left(d_{M}-1\right) G B\right) \frac{1}{\square_{0}} \\
& \times\left(\left.4 K\right|_{\theta^{2} \bar{\theta}^{2}}+\square_{0} K \mid\right)
\end{aligned}
$$

where $G B$ denotes the Gauss-Bonnet combination

$$
G B=C_{m n p q} C^{m n p q}-2 \mathcal{R}_{m n} \mathcal{R}^{m n}+\frac{2}{3} \mathcal{R}^{2}
$$

and we have not computed coefficient $d_{M}$ as it is irrelevant to the physics we wish to discuss. Note that the only Lorentz invariant gravitational combinations appearing in (6.3) are the square of the Weyl tensor, $C_{m n p q}$, and the Gauss-Bonnet combination $G B$. Also note that these are the only quadratic gravitational combinations that transform 
homogeneously under arbitrary Weyl-rescalings of the space-time metric $g_{m n}$. The fact that only these two combinations appear in (6.3) is a consequence of the conformal properties of the $\bar{A} A$ vertices of the graph shown in Figure 4b. Similarly, the fermion and scalar loop graphs of Figure 4c can be computed, and their contribution is found to be [19, 17]

$$
\mathcal{L}_{\chi, A}^{\prime}=\frac{1}{6 \cdot 24 \cdot(4 \pi)^{2}}\left(C_{m n p q} C^{m n p q}-\frac{1}{2} G B\right) \frac{1}{\square} \mathcal{R} .
$$

Once again, only the two gravitational combinations compatible with the conformal symmetry of the vertices appear in (6.5).

As before in the gauge case, the contribution of (6.5) to the renormalized trace of the energy momentum tensor can be evaluated using (5.8). We find that

$$
T^{\text {conf } m}{ }_{m}=-\frac{1}{24} \frac{1}{(4 \pi)^{2}}\left(C_{m n p q} C^{m n p q}-\frac{1}{2} G B\right)
$$

which is the well known gravitational contribution to the one-loop trace anomaly from conformal scalars and Weyl-fermions [19, 8]. Note that (6.6) can be put in the form

$$
T^{\text {conf } m}{ }_{m}=-\frac{1}{24} \frac{1}{(4 \pi)^{2}}\left(3 b C_{m n p q} C^{m n p q}-b^{\prime} G B\right)
$$

where

$$
\begin{aligned}
b & =\frac{1}{15}\left(N_{S}+3 N_{F}\right) \\
b^{\prime} & =\frac{1}{15}\left(N_{S}+\frac{11}{2} N_{F}\right)
\end{aligned}
$$

and $N_{S}(=2)$ is the number of scalars fields and $N_{F}(=1)$ is the number of Weyl fermions. Adding (6.1), (6.3) and (6.5) we find that

$$
\begin{aligned}
\mathcal{L}^{\text {conf }}= & -\frac{2 i \kappa^{2}}{3 \cdot 24 \cdot(4 \pi)^{2}} \mathcal{R}_{m n a}{ }^{b} \tilde{\mathcal{R}}^{m n}{ }_{b}{ }^{b} \frac{1}{\square_{0}} \partial^{p} c_{p} \\
& +\frac{1}{12 \cdot 24 \cdot(4 \pi)^{2}} C_{m n p q} C^{m n p q} \frac{1}{\square_{0}}\left(6 b \mathcal{R}+d_{M} \kappa^{2}\left[\left.4 K\right|_{\theta^{2} \bar{\theta}^{2}}+\square_{0} K \mid\right]\right) \\
& -\frac{1}{12 \cdot 24 \cdot(4 \pi)^{2}} G B \frac{1}{\square_{0}}\left(2 b^{\prime} \mathcal{R}+\left(d_{M}-1\right) \kappa^{2}\left[\left.4 K\right|_{\theta^{2} \bar{\theta}^{2}}+\square_{0} K \mid\right]\right)
\end{aligned}
$$

Using the identities [30

$$
\begin{array}{r}
\frac{1}{16}\left(C_{m n p q} C^{m n p q}-i \mathcal{R}_{m n a}{ }^{b} \tilde{\mathcal{R}}^{m n}{ }_{b}^{a}\right)=\left.W_{\alpha \beta \gamma}^{2}\right|_{\theta^{2}} \\
G B=\left.\left(8 W_{\alpha \beta \gamma}^{2}+\left(\overline{\mathcal{D}}^{2}-8 R\right)\left(G_{\alpha \dot{\alpha}}^{2}-4 R^{\dagger} R\right)\right)\right|_{\theta^{2}}+\text { h.c. }
\end{array}
$$


it follows that (6.9) is the component field expansion of the following superfield Lagrangian

$$
\begin{aligned}
\mathcal{L}^{\text {conf }} & =\frac{2}{3 \cdot(4 \pi)^{2}} \int d^{4} \theta W_{\alpha \beta \gamma}^{2} \frac{1}{\square_{0}}\left(\left(3 b-b^{\prime}\right) R^{\dagger}-\frac{\kappa^{2}}{24} D^{2} K\right) \\
& -\frac{1}{12 \cdot(4 \pi)^{2}} \int d^{4} \theta\left(\overline{\mathcal{D}}^{2}-8 R\right)\left(G_{\alpha \dot{\alpha}}^{2}-4 R^{\dagger} R\right) \frac{1}{\square_{0}}\left(b^{\prime} R^{\dagger}-\frac{\kappa^{2}}{24}\left(d_{M}-1\right) D^{2} K\right) \\
& +h c
\end{aligned}
$$

We conclude, then, that the one-loop three-point graphs shown in Figure 4a, b and c give rise to the supersymmetric effective Lagrangian (6.9), or, equivalently, to the superfield Lagrangian (6.11). Note that the background field dependent metric $g_{A A^{\dagger}}-$ $\frac{\kappa^{2}}{3} G_{A} G_{A^{\dagger}}$, which multiplies the $A \bar{A}$-vertices in (4.26), has dropped out in the effective Lagrangian (6.9). Hence, the anomaly coefficient in (6.11) is background field independent. Using (5.12), it follows that under super-Weyl-Kähler transformations

$$
\begin{aligned}
\delta \mathcal{L}^{\text {conf }} & =\frac{1}{72 \cdot(4 \pi)^{2}} \int d^{2} \theta\left(8\left(1+3 b-b^{\prime}\right) W_{\alpha \beta \gamma}^{2}\right. \\
& \left.-\left(d_{M}-1+b^{\prime}\right)\left(\overline{\mathcal{D}}^{2}-8 R\right)\left(G_{\alpha \dot{\alpha}}^{2}-4 R^{+} R\right)\right) F+\text { h.c. }
\end{aligned}
$$

which is non-vanishing. Hence, expression (6.11) represents the contribution to the supersymmetric mixed super-Weyl-Kähler-Lorentz anomaly from graphs constructed out of conformal vertices only.

Let us now turn to the contributions from triangle graphs constructed out of the conformal breaking $A^{2}$ and $\bar{A}^{2}$ vertices. The only possible graphs are shown in Figure $4 \mathrm{~d}$. These graphs contribute the following non-local term to the effective Lagrangian

$$
\begin{aligned}
\mathcal{L}^{\text {nonconf }}= & \frac{1}{12} \frac{1}{18} \frac{|\langle q\rangle|^{2}}{(4 \pi)^{2}}\left\{\left(\mathcal{R}-\frac{\kappa^{2}}{2}\left(\left.4 K\right|_{\theta^{2} \bar{\theta}^{2}}+\square_{0} K \mid\right)\right)^{2}\right. \\
& \left.\times \frac{1}{\square_{0}}\left(\mathcal{R}+\kappa^{2}\left(\left.4 K\right|_{\theta^{2} \bar{\theta}^{2}}+\square_{0} K \mid\right)\right)\right\}
\end{aligned}
$$

where $q$ is given by

$$
q=\frac{G_{A A}-\frac{\kappa^{2}}{3} G_{A} G_{A}}{g_{A A^{\dagger}}-\frac{\kappa^{2}}{3} G_{A} G_{A^{\dagger}}}
$$

and is the factor multiplying the $A^{2}$ vertex, as can be seen from (4.26). Factor $q$ is background field dependent, but invariant under Kähler transformations $\kappa^{2} K \rightarrow \kappa^{2} K+F+$ 
$\bar{F}$, since it is given in terms of the Kähler invariant combination $G=K+\ln W+\ln \bar{W}$. Note that it is not $q$, but the constant vacuum expectation value $\langle q\rangle$ that appears in (6.13). This occurs, because when calculating the anomalous contributions from the graphs discussed in this paper, one actually first expands the background field dependent metrics in Lagrangians (4.26) and (4.33) around constant vacuum expectation values $\langle A\rangle,\langle\bar{A}\rangle$. The $\left(\mathcal{R}-\frac{\kappa^{2}}{2}\left(\left.4 K\right|_{\theta^{2} \bar{\theta}^{2}}+\square_{0} K \mid\right)\right.$-legs in (6.13) arise through the coupling to the $A^{2}$ and $\bar{A}^{2}$ vertices, whereas the $\mathcal{R}+\kappa^{2}\left(\left.4 K\right|_{\theta^{2} \bar{\theta}^{2}}+\square_{0} K \mid\right)$-leg arises through the coupling to the $\bar{A} A$ vertex. Lagrangian (6.13) is obviously not invariant under superWeyl-Kähler transformations and, hence, anomalous. The component expression (6.13) is contained in the following superfield Lagrangian

$$
\begin{aligned}
\mathcal{L}^{\text {nonconf }}= & \frac{4|\langle q\rangle|^{2}}{(4 \pi)^{2}} \int d^{4} \theta \bar{D}^{2}\left\{\left(R^{\dagger}+\frac{\kappa^{2}}{24} D^{2} K\right)\left(R+\frac{\kappa^{2}}{24} \bar{D}^{2} K\right)\right\} \\
& \times \frac{1}{\square_{0}}\left(R^{\dagger}-\frac{\kappa^{2}}{12} D^{2} K\right)+\text { h.c. }
\end{aligned}
$$

Consequently, the total contribution to the mixed super-Weyl-Kähler-Lorentz anomaly is given by the sum of (6.11) and (6.15)

$$
\mathcal{L}^{\text {total }}=\mathcal{L}^{\text {conf }}+\mathcal{L}^{\text {nonconf }}
$$

We point out that expression (6.16) could also have been obtained by direct three-point supergraph calculations using the prepotential formalism [20, 9].

Lagrangian (6.16) represents the mixed super-Weyl-Kähler-Lorentz anomaly in conventional superspace. We would now like to transform it over into Kähler superspace. Following the manifestly supersymmetric procedure described at the end of section 5, we now apply the transformations (5.14) to the anomalous Lagrangian (6.16). The result is

$$
\begin{aligned}
\mathcal{L}^{\text {total }}= & \frac{1}{12(4 \pi)^{2}} \int d^{4} \theta\left(8\left(3 b-b^{\prime}\right) W_{\alpha \beta \gamma}^{2}-b^{\prime}\left(\overline{\mathcal{D}}^{2}-8 R\right)\left(G_{\alpha \dot{\alpha}}^{2}-4 R^{\dagger} R\right)\right) \frac{1}{\square_{0}} R^{\dagger} \\
& +\frac{4|\langle q\rangle|^{2}}{(4 \pi)^{2}} \int d^{4} \theta \bar{D}^{2}\left(R^{\dagger} R\right) \frac{1}{\square_{0}} R^{\dagger} \\
& -\frac{1}{24} \frac{\kappa^{2}}{(4 \pi)^{2}} \int d^{4} \theta\left(\frac{2}{3}\left(1+3 b-b^{\prime}\right) W_{\alpha \beta \gamma}^{2}\right. \\
& \left.-\frac{\left(d_{M}-1+b^{\prime}\right)}{12}\left(\overline{\mathcal{D}}^{2}-8 R\right)\left(G_{\alpha \dot{\alpha}}^{2}-4 R^{\dagger} R\right)\right) \frac{1}{\square_{0}} D^{2} K
\end{aligned}
$$




$$
-\frac{|\langle q\rangle|^{2}}{2} \frac{\kappa^{2}}{(4 \pi)^{2}} \int d^{4} \theta \bar{D}^{2}\left(R^{\dagger} R\right) \frac{1}{\square_{0}} D^{2} K+\text { h.c. }
$$

where the Lagrangian (6.17) is given in terms of Kähler superspace superfields $W_{\alpha \beta \gamma}$, $G_{\alpha \dot{\alpha}}, R, K$. The first two terms in (6.17) are invariant under Kähler transformations, as can be readily seen from (2.6). Hence, they can be discarded. The Kähler anomaly is entirely given by the last two terms

$$
\begin{aligned}
\mathcal{L}^{\text {anom }}= & -\frac{1}{24} \frac{\kappa^{2}}{(4 \pi)^{2}} \int d^{4} \theta\left(\frac{2}{3}\left(1+3 b-b^{\prime}\right) W_{\alpha \beta \gamma}^{2}\right. \\
& \left.-\frac{\left(d_{M}-1+b^{\prime}\right)}{12}\left(\overline{\mathcal{D}}^{2}-8 R\right)\left(G_{\alpha \dot{\alpha}}^{2}-4 R^{\dagger} R\right)\right) \frac{1}{\square_{0}} D^{2} K \\
& -\frac{|\langle q\rangle|^{2}}{2} \frac{\kappa^{2}}{(4 \pi)^{2}} \int d^{4} \theta \bar{D}^{2}\left(R^{\dagger} R\right) \frac{1}{\square_{0}} D^{2} K+\text { h.c. }
\end{aligned}
$$

These terms are obviously not invariant under Kähler transformations. Comparing the term proportional to $W_{\alpha \beta \gamma}^{2}$ in (6.18) with the minimal expression (3.7) shows that both agree! Lagrangian (6.18) thus qualifies to be called the supersymmetric Kähler-Lorentz anomaly in Kähler superspace. In component fields, the anomalous Lagrangian (6.16) reads

$$
\begin{aligned}
\mathcal{L}^{\text {anom }}= & -\frac{\kappa^{2}}{24 \cdot(4 \pi)^{2}} \mathcal{R}_{m n a}{ }^{b} \tilde{\mathcal{R}}^{m n}{ }_{b}^{a} \frac{1}{\square_{0}} \partial^{p} a_{p} \\
& +\frac{\kappa^{2}}{12 \cdot 24 \cdot(4 \pi)^{2}}\left(\left(d_{M}+3 b\right) C_{m n p q} C^{m n p q}-\left(d_{M}-1+b^{\prime}\right) G B\right) \frac{1}{\square_{0}} \\
& \times\left(\left[\left.4 K\right|_{\theta^{2} \bar{\theta}^{2}}+\square_{0} K \mid\right]\right) \\
& +\frac{1}{144} \frac{|\langle q\rangle|^{2} \kappa^{2}}{(4 \pi)^{2}} \mathcal{R}^{2} \frac{1}{\square_{0}}\left(\left.4 K\right|_{\theta^{2} \bar{\theta}^{2}}+\square_{0} K \mid\right)+\ldots
\end{aligned}
$$

where ... refers to terms containing fermions that we have not explicitly computed. Let us emphasize again that the coefficient $\langle q\rangle$ of the $\mathcal{R}^{2} K \mid$-piece in (6.19) is background field dependant. This is to be contrasted with the coefficients of the $C_{m n p q}^{2} K \mid$ - and $G B K \mid$-pieces in (6.19), which are background field independent.

Finally, we point out that the anomalies involving the sigma-model Christoffel connection can now easily be computed. This is done by replacing, in the conventional superspace calculation, $\kappa^{2} K$ by $\kappa^{2} K-3 \operatorname{lng}$ where $g$ is the Kähler metric for the chiral multiplet in the loop. Also, one can sum over all matter sectors, denoted by $\sum_{S}$, and over all matter multiplets, $n_{S}$, in a given sector. Furthermore, it is also not difficult to 
extend our results to include gauge vector multiplets running around the internal loop. The only changes are that the $b$ and $b^{\prime}$ coefficients are now to be evaluated for gauge vector multiplets, and the unknown coefficient $d_{M}$, which ocurred for chiral multiplets, here does not appear. Putting all of this together we find, after some manipulation, that

$$
\begin{aligned}
\mathcal{L}^{\text {anom }}= & -\frac{1}{36} \frac{\kappa^{2}}{(4 \pi)^{2}} \int d^{4} \theta\left(3 b W_{\alpha \beta \gamma}^{2}-\frac{b^{\prime}}{8}\left(8 W_{\alpha \beta \gamma}^{2}+\left(\overline{\mathcal{D}}^{2}-8 R\right)\left(G_{\alpha \dot{\alpha}}^{2}-4 R^{\dagger} R\right)\right)\right. \\
& \times \frac{1}{\square_{0}} D^{2} K \\
& +\frac{1}{24} \frac{1}{(4 \pi)^{2}} \int d^{4} \theta W_{\alpha \beta \gamma}^{2} \frac{1}{\square_{0}} D^{2}\left[\sum_{S}\left(2 \operatorname{lndetg}_{S}-\frac{2}{3} n_{S} \kappa^{2} K\right)\right] \\
& -\frac{1}{8 \cdot 24} \frac{1}{(4 \pi)^{2}} \int d^{4} \theta\left(\overline{\mathcal{D}}^{2}-8 R\right)\left(G_{\alpha \dot{\alpha}}^{2}-4 R^{\dagger} R\right) \\
& \times \frac{1}{\square_{0}} D^{2}\left[\sum_{S}\left(2 \ln \operatorname{det} g_{S}-\frac{2}{3} n_{S}\left(d_{M}-1\right) \kappa^{2} K\right)\right] \\
& +\frac{|\langle q\rangle|^{2}}{2} \frac{1}{(4 \pi)^{2}} \int d^{4} \theta \bar{D}^{2}\left(R^{\dagger} R\right) \frac{1}{\square_{0}} D^{2}\left[\sum_{S}\left(2 \operatorname{lndetg}_{S}-n_{S} \kappa^{2} K\right)\right] \\
& + \text { h.c. }
\end{aligned}
$$

where

$$
\begin{aligned}
b & =\frac{1}{15}\left(N_{S}+3 N_{F}+12 N_{V}\right) \\
b^{\prime} & =\frac{1}{15}\left(N_{S}+\frac{11}{2} N_{F}+62 N_{V}\right)
\end{aligned}
$$

are the trace anomaly coefficients summed over all matter and gauge vector supermultiplets. Note from (6.10) that the term proportional to $b$ contains in components a pure $\left(C_{m n p q}\right)^{2}$-term, whereas the term proportional to $b^{\prime}$ is the superfield expression containing the Gauss-Bonnet topological density. In component fields, the anomalous Lagrangian (6.20) reads

$$
\begin{aligned}
\mathcal{L}^{\text {anom }}= & -\frac{\kappa^{2}}{36 \cdot(4 \pi)^{2}}\left(3 b-b^{\prime}+N_{S}\right) \mathcal{R}_{m n a}{ }^{b} \tilde{\mathcal{R}}^{m n}{ }_{b} \frac{1}{\square_{0}} \partial^{p} a_{p} \\
& +\frac{\kappa^{2}}{12 \cdot 24 \cdot(4 \pi)^{2}}\left[\left(N_{S} d_{M}+3 b\right) C_{m n p q} C^{m n p q}-\left(N_{S} d_{M}-N_{S}+b^{\prime}\right) G B\right] \frac{1}{\square_{0}} \\
& \times\left(\left[\left.4 K\right|_{\theta^{2} \bar{\theta}^{2}}+\square_{0} K \mid\right]\right) \\
& +\frac{N_{S}}{144} \frac{|\langle q\rangle|^{2} \kappa^{2}}{(4 \pi)^{2}} \mathcal{R}^{2} \frac{1}{\square_{0}}\left(\left.4 K\right|_{\theta^{2} \bar{\theta}^{2}}+\square_{0} K \mid\right)+\ldots
\end{aligned}
$$


where, for simplicity, we have only displayed the terms proportional to $K$. Note from (6.21) that $3 b-b^{\prime}+N_{S}=\frac{3}{2}\left(N_{S}-N_{V}\right)$, which yields the correct coefficient in (6.22) multiplying the term proportional to $\mathcal{R} \tilde{\mathcal{R}}$.

We close this section with a brief comment on the relationship between the mixed supersymmetric Kähler-Lorentz anomaly, given by (6.22), and the moduli dependent threshold corrections to gravitational couplings [3] in $Z_{N}$ orbifolds. These corrections were computed for the gravitational coupling constant of $C_{m n p q}^{2}$. The coefficient of these corrections was found to be non vanishing for $Z_{N}$ orbifolds with $N=2$ spacetime sectors and to be proportional to the trace anomaly coefficients for the different fields coupled to gravity. This phenomena can now be somewhat understood in the field theory framework as follows. The coefficient of the $C_{m n p q}^{2} K \mid$-term in (6.22) has, as shown above, its origin in the coefficients of the two distinctive terms (6.3) and (6.5). The coefficient (6.3) is determined by the chiral anomaly (6.1) computed in the conventional superspace formulation, whereas the coefficient in (6.5) is given by the trace anomaly (6.6). By comparing against the results of the explicit string loop calculation of [3] it then follows that, in $Z_{N}$ orbifolds with $N=2$ space-time sectors, only a certain amount of the $C_{m n p q}^{2} K \mid$-term is removed by the Green-Schwarz mechanism [23]. This amount is given by the coefficient of (6.3), yielding a left-over proportional to (6.6), that is to the trace anomaly coefficients for the different fields coupled to gravity. For $Z_{N}$ orbifolds with no $N=2$ spacetime sectors, the entire amount given in (6.16) must be removed by the Green-Schwarz mechanism. This will be discussed in detail in another publication [13].

\section{Conclusion}

We have shown how to calculate the matter contributions to the mixed Kählergauge and mixed Kähler-Lorentz anomalies in a manifestly supersymmetric way. Even though we have restricted our analysis to matter loops only, the contributions from Yang-Mills and gravitational fields running in the loop can, in principle, also be computed along the same lines. All these contributions can be obtained by first performing calculations in the conventional formulation of supergravity-matter systems. In this formulation, there exists a prepotential superfield formalism which enables one to separate 
quantum degrees of freedom from background fields in a manifestly supersymmetric way. Calculations in this superfield formalism lead, by construction, to manifestly supersymmetric results. Such superfield calculations of matter loops should agree with the component field calculations presented above. All these results can be transformed over to the Kähler superspace formulation of the theory. In the latter formulation, the tree-level gravitational kinetic energy is appropriately Einstein normalised. The Kähler anomaly in Kähler superspace is obtained from the one in the conventional formulation by specific superfield rescalings of the underlying torsion constraints [7]. Since these redefinitions are defined at the superfield level, the resulting expression for the Kähler anomaly in Kähler superspace is manifestly supersymmetric.

We would like to emphasize the following. First of all, we have shown that the procedure described in this paper leads to the well known result for the mixed Kähler-gauge anomaly in Kähler superspace [16, 10, 26], which is responsible for non-holomorphic threshold corrections to the running of gauge couplings in string effective field theories 16, 10, 26, 18, 2, 3, 25. Secondly, the mixed Kähler-Lorentz anomaly contains, in general, a term proportional to the square of the curvature tensor, $\mathcal{R}^{2}$. Its coefficient, however, comes out to be background field dependent, in contrast to both the gauge case and to the terms in the mixed Kähler-Lorentz anomaly proportional to the square of the Weyl tensor, $C_{m n p q}^{2}$, and to the Gauss-Bonnet combination. As shown in [11, 12], a term in the Kähler anomaly proportional to $\mathcal{R}^{2}$ can drive inflation in the early universe and, hence, it can have very interesting cosmological consequences. Finally, some field theoretical understanding of the moduli dependent threshold corrections to gravitational couplings in $Z_{N}$ orbifolds [3] can be gained by comparing our results to the ones obtained in [3] by an explicit string loop calculation. These threshhold corrections, computed for the gravitational coupling of the $C_{m n p q}^{2}$-term, turn out to be proportional to the trace anomaly coefficients of the different fields coupled to gravity. From the field theory point of view this can be understood by noticing that there are two distinct terms which contribute to the Kähler anomaly, as described above. One of them comes with a coefficient proportional to the trace anomaly whereas the other one comes with a coefficient proportional to the chiral anomaly in conventional superspace. It is this latter contribution which gets removed by the Green-Schwarz mechanism 23, yielding threshold corrections proportional to the trace anomaly coefficients of the various fields 
coupled to gravity.

\section{References}

[1] L. Alvarez-Gaumé and P. Ginsparg, Annals of Phys. 161 (1985) 423.

[2] I. Antoniadis, K. S. Narain and T. R. Taylor, Phys. Lett. B267 (1991) 37.

[3] I. Antoniadis, E. Gava and K. S. Narain, Nucl. Phys. B383 (1992) 93.

[4] J. Bagger and E. Witten, Phys. Lett. B115 (1982) 202.

[5] P. Binétruy, G. Girardi, R. Grimm, and M. Müller, Phys. Lett. B189 (1987) 83.

[6] P. Binétruy, G. Girardi, R. Grimm and M. Müller, Phys. Lett. B195 (1987) 389.

[7] P. Binétruy, G. Girardi, and R. Grimm, LAPP-Preprint LAPP-TH-275/90.

[8] N. Birrell and P. Davies, Quantum Fields in Curved Spacetime (Cambridge Univ. Press 1982).

[9] G. L. Cardoso and B. A. Ovrut, Nucl. Phys. B369 (1992) 351.

[10] G. L. Cardoso and B. A. Ovrut, Nucl. Phys. B392 (1993) 315.

[11] G. L. Cardoso and B. A. Ovrut, Phys. Lett. B298 (1993) 292.

[12] G. L. Cardoso and B. A. Ovrut, CERN preprint CERN-TH.6847/93.

[13] G. L. Cardoso and B. A. Ovrut, CERN preprint (1993) in preparation.

[14] E. Cremmer, B. Julia, J. Scherk, S. Ferrara, L. Girardello and P. van Nieuwenhuizen, Phys. Lett. B79 (1978) 231; Nucl. Phys. B147 (1979) 105.

[15] E. Cremmer, S. Ferrara, L. Girardello and A. van Proyen, Phys. Lett. B116 (1982) 231; Nucl. Phys. B212 (1983) 413. 
[16] J. P. Derendinger, S. Ferrara, C. Kounnas and F. Zwirner, Nucl. Phys. B372 (1992) 145.

[17] S. Deser, M. Duff and C. Isham, Nucl. Phys. B111 (1976) 45.

[18] L. J. Dixon, V. S. Kaplunovsky and J. Louis, Nucl. Phys. B355 (1991) 649.

[19] M. Duff, Nucl. Phys. B125 (1977) 334.

[20] S. J. Gates, K. T. Grisaru, M. Roček and W. Siegel, Superspace (Benjamin Cummings, Reading, PA, 1983).

[21] G. Girardi and R. Grimm, Phys. Lett. B260 (1991) 365.

[22] R. Grimm, J. Wess and B. Zumino, Nucl. Phys. B152 (1979) 255.

[23] M. B. Green and J. H. Schwarz, Phys. Lett. B149B (1984) 117.

[24] P. S. Howe and R. W. Tucker, Phys. Lett. B80 (1978) 138.

[25] L. E. Ibánez and D. Lüst, Nucl. Phys. B382 (1992) 305.

[26] J. Louis, PASCOS 1991 Proceedings, P. Nath ed., World Scientific 1991.

[27] G. Moore and P. Nelson, Phys. Rev. Lett. 53 (1984) 1519.

[28] M. Müller, Nucl. Phys. B264 (1986) 292.

[29] M. A. Shifman and A. I. Vainshtein, Phys. Scr. T 15 (1987) 184.

[30] S. Theisen, Nucl. Phys. B263 (1986) 687.

[31] J. Wess and J. Bagger, Supersymmetry and Supergravity, Second Edition (Princeton University Press, 1990). 


\section{Figure Captions}

Figure 1a: Fermionic contribution to the mixed Kähler-gauge anomaly.

Figure 1b: Fermionic contribution to the mixed Kähler-Lorentz anomaly.

Figure 2a: Fermionic contribution to the mixed super-Weyl-Kähler- gauge anomaly.

Figure 2b: Scalar contribution to the mixed super-Weyl-Kähler- gauge anomaly.

Figure 2c: Fermionic and scalar contributions to the mixed super-Weyl-Kähler- gauge anomaly.

Figure 3: The gauge two-point function.

Figure 4a: Fermionic contribution to the mixed super-Weyl-Kähler- Lorentz anomaly. Figure 4b: Scalar contribution to the mixed super-Weyl-Kähler- Lorentz anomaly constructed from conformal vertices, only.

Figure 4c: Fermionic and scalar contributions to the mixed super-Weyl-Lorentz anomaly constructed from conformal vertices, only.

Figure 4d: Graphs contributing to the mixed super-Weyl-Kähler-Lorentz anomaly constructed from non-conformal vertices. 